\title{
A Survey on the Role of Centrality as Seed Nodes for Information Propagation in Large Scale Network
}

\author{
PARAMITA DEY, Government College of Engineering \& Ceramic Technology, India \\ SUBHAYAN BHATTACHARYA and SARBANI ROY, Jadavpur University, India
}

\begin{abstract}
From the popular concept of six-degree separation, social networks are generally analyzed in the perspective of small world networks where centrality of nodes play a pivotal role in information propagation. However working with a large dataset of a scale-free network (which follows power law) may be different due to the nature of the social graph. Moreover, the derivation of centrality may be difficult due to the computational complexity of identifying centrality measures. This study provides a comprehensive and extensive review and comparison of seven centrality measures (clustering coefficients, Node degree, K-core, Betweenness, Closeness, Eigenvector, PageRank) using four information propagation methods (Breadth First Search, Random Walk, Susceptible-Infected-Removed, Forest Fire). Five benchmark similarity measures (Tanimoto, Hamming, Dice, Sorensen, Jaccard) have been used to measure the similarity between the seed nodes identified using the centrality measures with actual source seeds derived through Google's LargeStar-SmallStar algorithm on Twitter Stream Data. MapReduce has been utilized for identifying the seed nodes based on centrality measures and for information propagation simulation. It is observed that most of the centrality measures perform well compared to the actual source in the initial stage but are saturated after a certain level of influence maximization in terms of both affected nodes and propagation level.
\end{abstract}

CCS Concepts: • Human-centered computing $\rightarrow$ Empirical studies in collaborative and social computing; Social networks; Social recommendation; • Social and professional topics $\rightarrow$ User characteristics;

Additional Key Words and Phrases: Large scale social network, Influence maximization, Centrality measures, Information propagation, Similarity measures

ACM Reference format:

Paramita Dey, Subhayan Bhattacharya, and Sarbani Roy. 2021. A Survey on the Role of Centrality as Seed Nodes for Information Propagation in Large Scale Network. ACM/IMS Trans. Data Sci. 2, 3, Article 24 (August 2021), 25 pages.

https://doi.org/10.1145/3465374

\footnotetext{
Authors' addresses: P. Dey, Government College of Engineering \& Ceramic Technology, 73, A. C. Banerjee Lane, Kolkata700010, India; emails: Paramitadeyt@gmail.com, paramitadey@gcect.ac.in; S. Bhattacharya, Jadavpur University, 188, Raja S. C. Mullick Road, Kolkata-700032, India; email: b.subhayan@yahoo.com; S. Roy, Jadavpur University, Department of Computer Science \& Engineering, 188, Raja S. C. Mullick Road, Kolkata-700032, India; email: sarbani.roy@jadavpuruniversity.in. Permission to make digital or hard copies of all or part of this work for personal or classroom use is granted without fee provided that copies are not made or distributed for profit or commercial advantage and that copies bear this notice and the full citation on the first page. Copyrights for components of this work owned by others than ACM must be honored. Abstracting with credit is permitted. To copy otherwise, or republish, to post on servers or to redistribute to lists, requires prior specific permission and/or a fee. Request permissions from permissions@acm.org. (C) 2021 Association for Computing Machinery.

2577-3224/2021/08-ART24 \$15.00

https://doi.org/10.1145/3465374
}

ACM/IMS Transactions on Data Science, Vol. 2, No. 3, Article 24. Publication date: August 2021. 


\section{INTRODUCTION}

\subsection{Overview}

Online social networks (OSNs) act as an important medium of information propagation and influence maximization in modern society. The spread of information, and influence, through OSNs can in turn influence real-life phenomenons, such as viral marketing, flash mobs, and unified protests. Information propagation and influence maximization also help in understanding public opinion, like opinion formation in Brexit [36], or U.S. presidential polls through social platforms like Facebook and Twitter. Thus, information propagation and influence maximization are the key areas of interest in recent research.

A crucial starting point for information propagation is seed node selection. The seed nodes are considered to be the source of the information. The more influential the seed node, the higher the information spread [10]. There is, however, an alternative line of thought that seed node selection is not related to information propagation in any way [17]. However, there is much research literature in support of significance of seed nodes in information spread.

Different centrality measures, such as clustering coefficients, Node degree, Betweenness, Closeness, Eigenvector, K-core, and PageRank, can be used to identify seed nodes in an OSN. Over the years, several measures of centrality have been proposed by different researchers [13]. For example, if an OSN is modeled as a graph $G(V, E)$, where $V$ denotes the set of vertices representing the users and $E$ denotes the edge set, then higher node degree centrality could signify higher influence. However, it can happen that it inherits the properties of local maxima where influence is confined within the local community. For such reasons, there is no universal consent on which measure is appropriate for influence maximization. Multiple information propagation methods, such as Breadth First Search, Random Walk, Susceptible-Infected-Removed (SIR), and Forest Fire, have been considered to preserve generalization.

Evidence exists in the literature that centrality measure plays a major role as a seed node when a network shows the small world network phenomenon [37]. This article focuses on establishing the same for large-scale networks.

For the purpose of this work, the Twitter data stream has been considered, Twitter, which is a very popular OSN site, gives the option of opinion generation. One can generate a maximum of 280 characters (previously 140 characters) as a tweet and others can share (retweet) on that tweet information.

\subsection{Contribution}

In this research work, a thorough literature review and an extensive experimental study has been conducted for the derivation of the following:

(i) Role of centrality measures as seed nodes for propagation

(ii) Similarity between centrality measures and the true seed node from where the information is actually generated using five benchmark similarity measures (namely, Tanimoto, Hamming, Dice, Sorensen, and Jaccard)

(iii) Comparative study between different centrality measures as the seed node of the propagation.

\subsection{Organization of the Article}

The rest of the article is organized as follows. Literature review and background are discussed in Section 2. Section 3 presents the problem statement and proposed approach to study the influence of centrality measures as seed nodes in the OSN. The implementation in the Spark framework 
is discussed in Section 3.5. Results and analysis are presented in Section 4. Finally, the article is concluded in Section 5.

\section{LITERATURE REVIEW AND BACKGROUND}

OSNs are generated by nodes, representing the user in the network, and edges, representing the interaction patterns among the users [7]. Recent research depicts the fact that the topology generated from the user interaction patterns inherit centrality patterns within the network $[2,15]$. A challenging motivation in network science is to find the relationship between the structure of a network, the dynamic change of the structure, and the influence of the nodes within the network, and to find contagion of information [28]. Using the structural properties of the network, the information flow in the network can be controlled $[6,23]$ using these methodologies.

\subsection{Influence Maximization}

Different controlling factors [16] are responsible for the maximization of information flow in the network $[12,38]$. Due to their topological advantages, centrality nodes are generally more influential than others in the network as compared to other nodes present in the network, and it is expected that this kind of node can act as spreader in the network for maximizing the influence [34]. Arruda et al. [10], in their research work, show that K-shell decomposition can be used to identify the most influential spreaders in the network. This K-core nodes represent the core of the network and may not be the most connected nodes in the network. An earlier work by Dey et al. [31] show the effect of influential node selection on influence maximization as well. Another recent work by Chen and Teng [5] focuses on studying the connection between static properties such as node significance centrality and the dynamic influence process in an OSN.

Holthoefer and Moreno [17] claim that influential spreader identification is not possible using the same parameters for standard information propagation models, and show that spread from K-core centrality make no significant difference in information propagation. Although most researchers claim that centrality nodes can be utilized as an influential spreader, the result is not uniform. In some recent research work, it was shown that some centrality measures can distinguish nodes with better granularity, whereas for some centrality measures, the performance is poor [14]. Some researchers generate a greedy strategy to influence the maximum number of users for spreading the information [20]. In the work of Sun and Tang [33], a comparative study of different influence maximization algorithms is executed.

\subsection{Network Modeling}

OSNs like Facebook and Twitter generate large-scale data and exhibit the properties of scale-free networks [26]. The Erdos-Renyi random graph model [9] can be used to simulate a complex realworld network, but the dynamic nature of the real-world graph cannot be characterized through this model. The Barbasi-Albert model [1], however, follows the power law (i.e., node degree distribution follows the power law) and can be used to simulate the structure of a scale-free network. The scale-free network can be considered as a collection of small networks whose center is called a hub. A small world model was proposed by Watts and Strogatz [37], based on the small world criterion (stated as six-degree separation) where clustering coefficient is much more as compared to a random network with the same number of nodes. This theory stated that all nodes are connected friends of friend. Therefore diameter of the network is much less as compared to the number of nodes in the network. 


\subsection{Centrality Measures}

Centrality measures identify nodes of importance in a given graph of the form $G(V, E)$. There are multiple centrality measures, and each one considers specific concepts and confronts the other. For example, Betweenness and Closeness centrality are derived from all possible shortest paths calculated from the network [11], ignoring existing alternative paths between them. Rusinowska et al. [32] show the effect of centrality measures on influence of nodes and propose to model this using a relation-algebraic approach. A recent work by Li [24] focuses on one particular centrality measure, namely Betweenness centrality, and its effect on the Bulletin Board Server.

\subsection{Propagation Approaches}

There exist several propagation models based on different diffusion models used in the network. The most popular approach is Random Walk, and its variants like Markov chain Monte Carlo Random Walk, where the walk is done from the seed node with a predefined probability. Viana et al. [35] propose a model of thermal diffusion to spread the information. There exist several probabilistic approaches for simulating the spreading of the information in the network. Zhang et al. [22] propose a new information dissemination model based on individual behavior that includes the visibility of content on a social network under constraints like time. A new contextspecific framework for recommending and diffusing education resources in a social network is proposed by Neves et al. [27]. Dey et al. [30] provide a survey on different information propagation approaches in a small world network.

To increase efficiency of calculation of centrality measures for large graphs and information diffusion in large graphs, distributed and parallel implementation of classic graph theory algorithms have also been proposed in the literature. Betweenness centrality is a computationally complex centrality measure, and Jamour et al. [18] suggest a parallel algorithm for it. Jo et al. [19] provide a fast and scalable distributed implementation of the Loopy Belief Propagation method to fit the huge volume of real-world graphs.

Table 1 provides a representation of the taxonomy of recent popular work in relevant and related topics. This article focuses on combining all three aspects-that is, centrality measures, information propagation, and distributed implementation-and does a comprehensive study on influence maximization through choice of seed nodes using centrality measures using multiple information propagation models, and utilizes distributed implementation for efficiency without compromising accuracy.

\section{PROPOSED SOLUTION}

\subsection{Problem Statement}

Given a graph $G(V, E), C \in\{$ Node degree, Betweenness, Closeness, Eigenvector, clustering coefficient, K-core, PageRank $\}$ denotes set of centrality measures. For $A \in\{$ Breadth First Search, Random Walk, Susceptible-Infected-Removed, Forest Fire\}, a set of information propagation algorithms, and $M \in\{$ Tanimoto, Hamming,Dice, Sorensen, Jaccard $\}$, a set of similarity measures, a set of seed nodes $S$ using $C$ is derived such that the set of infected nodes $I$ is obtained. Using $a \in A$ similarity is measured using $m \in M$ in the original set of infected nodes $I_{\text {original }}$ with $I$.

\subsection{Centrality Measure}

For the generation of influential source of propagation, seven centrality measures (Node degree centrality, Betweenness centrality, Closeness centrality, clustering coefficient, Eigenvector centrality, K-core, and PageRank) have been considered. Properties of these centrality measures are briefly described next. 
Table 1. Taxonomy of Recent Popular Work in Related Topics

\begin{tabular}{|l|l|}
\hline Author & \multicolumn{1}{c|}{ Cescription } \\
\hline \multicolumn{2}{|l|}{ Centrality Measures and Influence } \\
\hline Li, 2018 [24] & Centrality analysis of OSN data \\
\hline Chen \& Teng, 2017 [5] & Interplay between social influence and network centrality \\
\hline Grando et al. 2016 [14] & Compute vertex centrality in a complex massive social network \\
\hline Arruda et al., 2014 [10] & Describe role of centrality as an influential spreader \\
\hline Holthoefer \& Moreno, 2012 [17] & Absence of an influential spreader in a social network \\
\hline Rusinowska et al., 2011 [32] & Describe centrality and its influence \\
\hline Sun \& Tang, 2011 [33] & Social influence analysis \\
\hline Brandes, 2008 [3] & Betweenness centrality in a complex network and computation \\
\hline Newman, 2002 [28] & Describe different types of centrality measures \\
\hline \multicolumn{2}{|l|}{ Information Propagation Model } \\
\hline Neves et al., 2019 [27] & Diffusion model of information in an education network \\
\hline Zhang et al., 2017 [22] & Information propagation model based on individual behavior \\
\hline \multicolumn{2}{|c|}{ Distributed and Parallel Implementation } \\
\hline Jo et al., 2018 [19] & Fast and Scalable Distributed Loopy Belief Propagation \\
\hline Jamour et al., 2018 [18] & Parallel Algorithm for Incremental Betweenness Centrality on Large Graphs \\
\hline \hline
\end{tabular}

3.2.1 Node Degree Centrality. The degree centrality of a node $n \in V$ is defined by $D_{C}(n ; G)=$ degree $(n) /(V-1)$, where degree $(v)=|\{u \mid(v, u),(u, v) \in E\}|$. The Node degree [16] informs the number of connections formed among the nodes in the network. It is not indicative of frequency of communication and often is a local maxima with respect to the network.

3.2.2 Betweenness Centrality. The Betweenness centrality of a node $n \in V$ is defined by between $(n)=\sum_{s \neq n \neq t} \frac{\sigma_{s t}(n)}{\sigma_{s t}}$, where $\sigma_{s t}$ is the total number of shortest paths from node $s$ to node $t$ and $\sigma_{s t}(n)$ is the number of those that pass through $n$. The Betweenness centrality [16] indicates the capability of a node for faster transfer of information through the network.

3.2.3 Closeness Centrality. The closeness of a node $n \in V$ can be defined as $\operatorname{close}(n)=$ $\frac{1}{\sum_{v \in V} \delta(n, v)}$, where $\delta(n, v)$ is the shortest distance from $n$ to $v$. The Closeness centrality [16] indicates how fast information can spread from the node to the rest of the network. Note that if any node is $v$ is unreachable from the node $n$, then $\operatorname{close}(n)=0$. To solve this, mean harmonic distance can be used as $\frac{1}{|V|-1} \sum_{v \neq n \in V} \frac{1}{\delta(n, v)}$.

3.2.4 Clustering Coefficient. Clustering coefficient for a node $n \in V$ is defined as $\operatorname{cluster}(n)=$ $\frac{\lambda(n)}{\tau(n)}$, where $\lambda(n)$ represents the number of triangles and $\tau(n)$ represents the number of triples containing $n$. It is a measure of the degree to which nodes in a graph tend to cluster together [16].

3.2.5 K-core. A K-core of a graph $G$ is a maximal connected subgraph of $G$ in which all vertices have degree at least $K$. Equivalently, it is one of the connected components of the subgraph of $G$ formed by repeatedly deleting all vertices of degree $<K$ [16]. In a graph $G=(V, E)$ having $|V|=n$ and $|E|=m$, a subgraph $G^{\prime}=\left(V^{\prime}, E^{\prime}\right)$ formed out of $G$ is called a $K$-core if and only if $\operatorname{deg}(v) \geq K, \forall v \in V^{\prime}$, and for a preassigned positive integer $k$, moreover $G^{\prime}$ is maximal in the sense that it cannot be augmented further without destroying the property of K-core. A node belonging to K-core but not including a $(k+1)$ core is assigned K-core centrality $K$.

3.2.6 Eigenvector Centrality. Eigenvector centrality is a measure of the degree of influence of a node in a network that is calculated as a function of the influence of its neighbors. It can be formulated as eigen $(x)=1 / \lambda \sum x_{t} \forall t \in N(x)$ where $N(x)$ is the neighbors of $x$ in the network and $x_{t}$ is the weight of the edge $(x, t)[16]$. 
3.2.7 PageRank. PageRank [29] is a link analysis algorithm, and it assigns a numerical weighting to each node in a graph, with the purpose of measuring its relative importance within the set. The PageRank of a node $n \in V$ is defined as $\operatorname{prank}(n)=\frac{1-d}{|V|}+d \sum_{v:(v, n) \in E} \frac{\operatorname{prank}(u)}{|\{v:(v, u) \in E\}|}$, where $d$ is a dampening factor.

\subsection{Propagation Models}

For the purpose of this research work, four propagation models have been considered: Breadth First Search, Random Walk, SIR, and Forest Fire. Details of these models are provided next.

3.3.1 Forest Fire. The Forest Fire model was first presented by Bak et al. [39], where propagation emulates the pattern a fire grows in the forest [8]. The fire is started from the seed node, and concept is that the nodes close to the affected nodes will be affected faster than other nodes. The infection will be spread in a manner similar to the fire spread in a forest extended from a single tree.

3.3.2 Breadth First Search. In Breadth First Search, propagation depends on the probabilistic searching techniques considering seed nodes as root nodes [8]. In the proposed approach, the probability is considered as 1 , and therefore if a node is connected with an affected node, then for the next level the nodes will be affected through the information.

3.3.3 Random Walk. For Random Walk, propagation of the node follows a path that is randomly selected from the seed node [8]. The concept of the propagation is that if some nodes are connected with the affected nodes, the affected node will choose a node between them randomly to spread the information in the next level.

3.3.4 SIR Model. In the SIR model, a portion of the nodes is considered to be infected. The rest of the nodes are considered to be susceptible. Infected nodes can infect susceptible nodes. Infected nodes can also recover, in which case it can be labeled as recovered. Once recovered, nodes cannot go back to the susceptible state. The rate of infection is given based on a parameter beta, and the rate of recovery is given based on a parameter lambda [30].

\subsection{Proposed Methodology}

Information can be defined as the set of all tweets containing a particular \#hashtag. But the network generated from all nodes participating in the tweet generation will have a large volume to process. Moreover, the network will be sparse in nature, which will contain a large number of unnecessary data. Therefore, we need to segregate those nodes that are actively participating in \#hashtag generation.

Nodes, which signify users in a network, can be classified according to their nature of participation in the network:

- Source: A user who originates the information for a particular flow.

- Propagator: A user who does not originate the information but knows about and propagates it.

- Stifler or Sink: A user who knows about the information but does not propagate the information (i.e., the flow ends at a sink). This usually happens in two situations:

- The user is unwilling to share the information.

- The information loses its significance with time.

- Ignorant: A user who does not know about the information.

In broad sense, nodes can be grouped into two types of nodes: active (Source/Propagator or Spreader) or inactive (Stifler/Ignorant). For the network formation, only active nodes are considered. To study the propagation of information on Twitter data, a directed Tweet Information Graph 


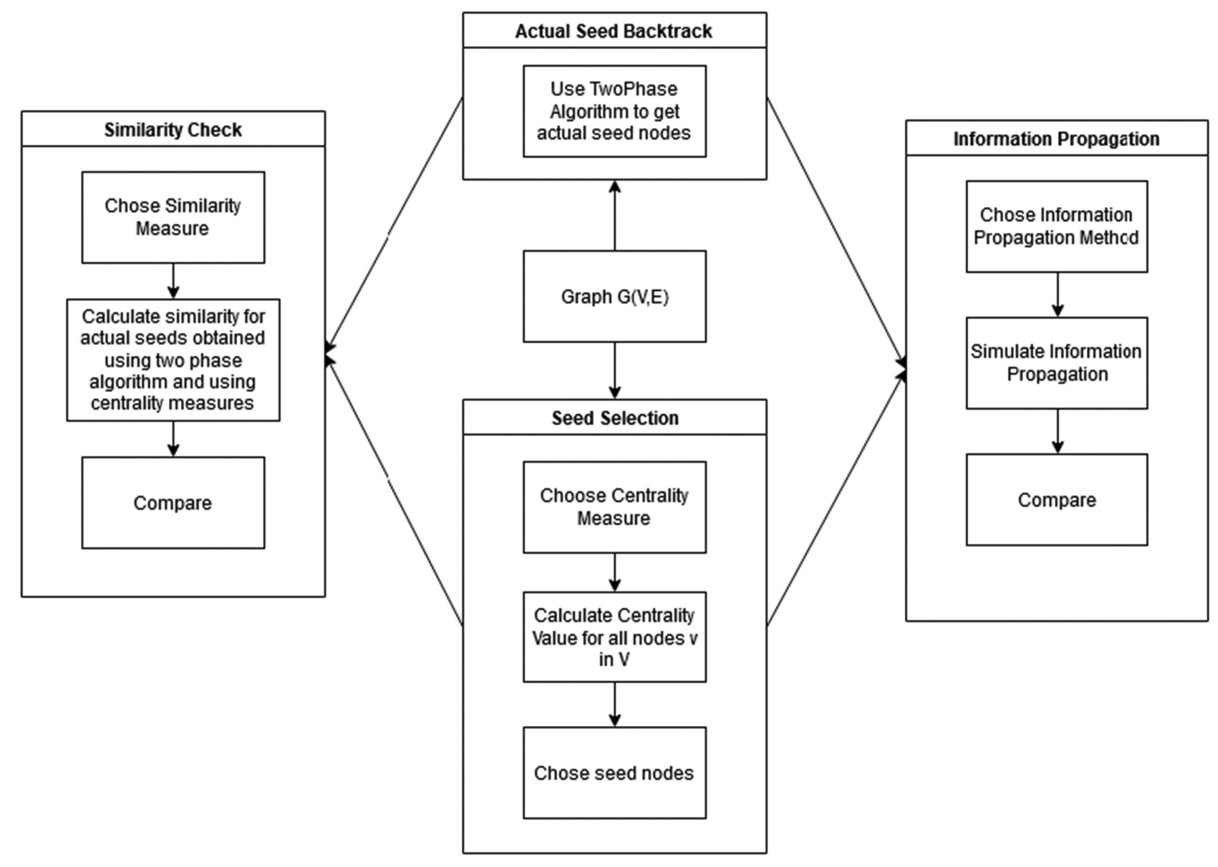

Fig. 1. Schematic Representation of Proposed Approach.

is defined with each node representing a user in the network. Nodes are active or inactive, based on whether their corresponding users are active or inactive participants. All active nodes are labeled with the timestamp of their first tweet containing the specified \#hashtag. All inactive nodes are ignored. The edge set consists of connections between nodes satisfying the following:

- Both nodes are active.

- There exists a Following link between the two corresponding nodes in the Twitter User Graph.

- The followee has a lower timestamp than the follower.

Here, the followee signifies those "who are followed by the user," and the follower signifies those "who follow the user."

The nodes selected through different centrality measures as discussed in Section 3.2 are considered as influential nodes and considered for the source of propagation in the Tweet Information Graph. These nodes are termed as influential seeds in the proposed methodology. The spread of information in the Tweet Information Graph is simulated using the propagation models discussed in Section 3.3. A schematic diagram representing the proposed method is shown in Figure 1.

True seeds are generated using the Google two-phase Largestar-Smallstar algorithm, and propagation is measured using the same algorithms and the results are compared.

\subsection{Distributed Implementation}

OSNs produce a huge volume of data at a high velocity. Standard graph-theoretic algorithms are not sufficient for producing acceptable results with high efficiency. Thus, the MapReduce approach is chosen to handle the high volume of data. For the purpose of this research, the Spark platform has been utilized. 
Table 2. Comparison Between Spark and Some of the Other Distributed Big Data Tools

\begin{tabular}{c|c}
\hline \multicolumn{2}{c}{ Spark vs. MapReduce } \\
\hline $\begin{array}{c}\text { In-RAM computation } \\
\text { Faster compared to MapReduce } \\
\text { More suited for data that can fit into RAM }\end{array}$ & $\begin{array}{c}\text { Required dedicated file system } \\
\text { Slower compared to Spark }\end{array}$ \\
\hline \multicolumn{2}{|c}{ Spark vs. Hive } \\
\hline \hline $\begin{array}{c}\text { Compatible with Python, Scala, Java } \\
\text { Independent of any framework }\end{array}$ & $\begin{array}{c}\text { Mostly works with Java } \\
\text { Uses Hadoop framwork }\end{array}$ \\
\hline \hline & Spark vs Cassandra \\
\hline Data Analysis Tool & High availability and scalability \\
Faster computation & Database \\
\hline
\end{tabular}

3.5.1 Spark. Spark excels in applications that exhibit cyclic dataflow models, or reuse a working set of data across multiple parallel operations, while retaining the scalability and fault tolerance of MapReduce [25]. MapReduce is a paradigm for distributed computation where Map with the input data generates another set of data in (key, data) format and distributes to different resources for computation and in reducer all results are compiled from the mapper to generate the output. In the Spark framework, both Map and Reduce can be generated in a single instance. The other alternative to Spark is Hadoop MapReduce, which is also a Big Data Analytics tool. However, Spark has some considerable advantages over Hadoop, ${ }^{1,2}$ such as Spark works In-Memory, whereas Hadoop requires a dedicated File System, commonly known as Hadoop File System (HDFS), which makes Spark more suitable for datasets that can be fitted into the RAM. Spark is also potentially a lot faster compared to Hadoop MapReduce. Spark also reduces complexity compared to the two-stage paradigm maintained by Hadoop MapReduce. In comparison to Hive as well, Spark has certain benefits that cannot be overlooked. ${ }^{3}$ Hive utilizes the underlying Hadoop framework. Spark is more compatible with a larger number of programming languages, such as Python and Scala. Similarly, in between Cassandra and Spark ${ }^{4}$ as well, Spark seems to be a better-suited option for the problem at hand. Thus, Spark has been considered for the distributed implementation of the algorithmic approach described in this article.

3.5.2 Algorithm Formulation. The graph-theoretic algorithms are all popular algorithms that have been reused here. However, these algorithms, without any adaptations, are not sufficient to process the huge volume of data produced in real-life OSNs. That is why an adaptation of the existing algorithms for the distributed implementation has been utilized for the purpose of this work. This allows the computation of computationally complex centrality measures such as Betweenness centrality possible over the whole network.

The general approach can be divided into two modules. The first module, which will be referred to as the iteration step, maps each node to its initial value and performs some filtration based on additional parameters. In the algorithmic representation 1, two filtration steps have been considered. The first filtration is applied on the complete graph. Then, for the filtered graph, each node is mapped to its initial value. The second filtration step then processes the nodes/edges based on the initial values of the nodes. During application, either or both of these filtration steps might

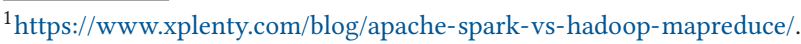

${ }^{2}$ https://logz.io/blog/hadoop-vs-spark/.

${ }^{3}$ https://www.educba.com/apache-hive-vs-apache-spark-sql/.

${ }^{4}$ https://stackshare.io/stackups/cassandra-vs-spark.
} 
be present. For example, in $K$-core, given two nodes $u$ and $v$, the iteration step first filters out all edges not containing either $u$ or $v$. For each node, the out degree is then calculated. The edges are retained if the out degree of the source is greater than $K$.

The second module, which will be referred to as the convergence step, utilizes the iteration step until the values converge. A state of convergence can be said to have been achieved if for two consecutive iterations the results are the exact same. The convergence step accepts as input the initial graph and then calls the iteration step. The initial graph is updated with the results of the iteration step. This is repeated until a state of convergence is achieved. For example, in $K$ core, the convergence step continues until no new edges/nodes can be removed from the graph. A general approach is pointed out in Algorithm 1 and Algorithm 2.
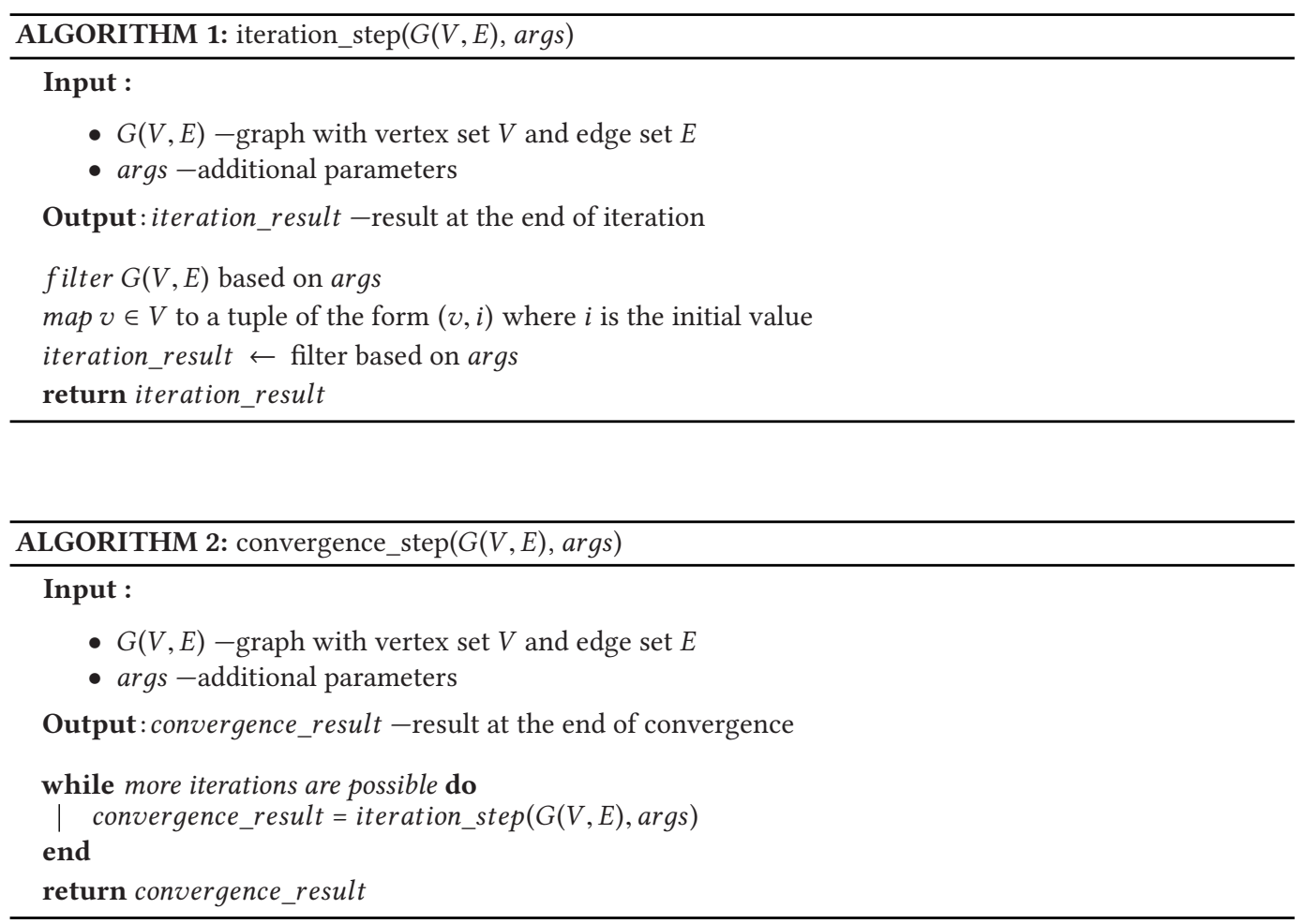

\section{EXPERIMENTAL OUTCOME}

\subsection{Dataset}

For the purpose of this work, six datasets have been collected. The user graph is derived from the 2009 database of Kwak et al. [21]. ${ }^{5}$ The open source dataset was collected from archive.org. ${ }^{6}$ A distribution diagram of collected tweets, which is in the range of a million $\left(10^{6}\right)$, is shown in Figure 2. Five other hashtags were collected from Twitter data streams using a filter query using the keywords \#covid, \#pandemic, \#quarantine, \#lockdown, and \#mask. All of these keywords are associated with the recent outbreak of global pandemic Covid19 and are now trending in OSNs.

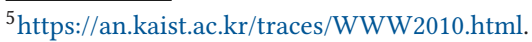

${ }^{6}$ https://archive.org/details/twitterstream (using the hashtag \#Brexit).
} 


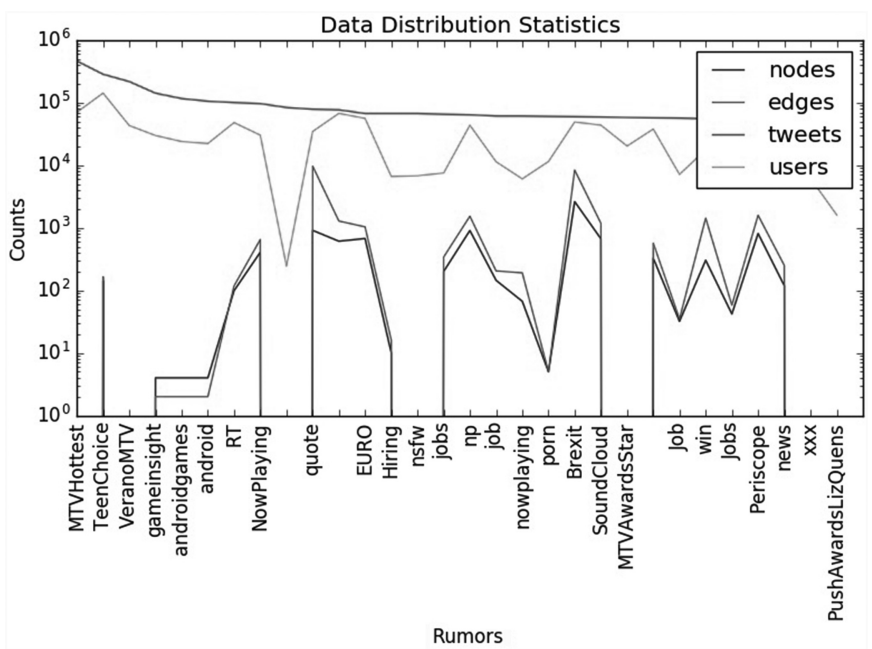

Fig. 2. Twitter dataset distribution statistics.

Table 3. Similarity Measures

\begin{tabular}{|c|c|}
\hline $\operatorname{Jaccard}\left(S_{1}, S_{2}\right)$ & $\frac{\left|S_{1} \cap S_{2}\right|}{\left|S_{1} \cup S_{2}\right|}$ \\
$\operatorname{Dice}\left(S_{1}, S_{2}\right)$ & $\frac{\left|S_{1} \cap S_{2}\right|}{\left|S_{1} \cap S_{2}\right|+\frac{1}{2}\left(\left|S_{1}-S_{2}\right|+\left|S_{2}-S_{1}\right|\right)}$ \\
$\operatorname{Tanimoto}\left(S_{1}, S_{2}\right)$ & $\frac{\left|S_{1} \cap S_{2}\right|}{\left|S_{1} \cap S_{2}\right|+\left|S_{1}-S_{2}\right|+\left|S_{2}-S_{1}\right|}$ \\
$\operatorname{Sorensen}\left(S_{1}, S_{2}\right)$ & $2 \frac{\left|S_{1} \cap S_{2}\right|}{\left|S_{1}\right|+\left|S_{2}\right|}$ \\
$\operatorname{Hamming}\left(S_{1}, S_{2}\right)$ & $\frac{\left|S_{1} \cap S_{2}\right|}{\max \left(\left|S_{1}\right|,\left|S_{2}\right|\right)}$ \\
\hline
\end{tabular}

The preceding datasets were chosen for several reasons. First, they aptly represent the data rate for OSNs, where a huge amount of data is generated over a short period of time. The size of the datasets also make them ideal to be processed over distributed implementation. The datasets are open source, and thus can be re-evaluated and re-used for other scopes on work and to build on this existing work. In addition, the datasets were collected over hashtags that are both relevant at the time of experimentation and on the longer run as well.

\subsection{Influential Seed Selection}

In this section, the success of centrality measures as influential seed detection algorithms is measured against the true seeds derived from the data. Table 3 represents five similarity measures [4] and the equations for calculating the corresponding similarity measures.

A comparison of the similarity between actual and influential seeds, with respect to different measures, for various centrality measurement algorithms is shown in Table 4 for the datasets. Here, actual seed indicates the source of actual information and influential seed indicates the node with the highest centrality value. The set of influential seeds was obtained each time by first ranking all nodes in the graph using the centrality measure, then selecting the nodes corresponding to the top $10 \%, 20 \%$, and $50 \%$, respectively. The values in the table represent the similarity values. 
Table 4. Comparison of the Similarity Between Actual and Highest Centrality Seeds: D-Dice, T-Tanimoto, H-Hamming, Jaccard, and Sorensen Results Coincide with Tanimoto and Dice, Respectively

\begin{tabular}{|c|c|c|c|c|c|c|c|c|c|c|}
\hline \multirow{2}{*}{ Experiment } & \multirow{2}{*}{ Properties } & \multicolumn{3}{|c|}{$10 \%$} & \multicolumn{3}{|c|}{$20 \%$} & \multicolumn{3}{|c|}{$50 \%$} \\
\hline & & $D$ & $T$ & $H$ & $D$ & $T$ & $H$ & $D$ & $T$ & $H$ \\
\hline \multirow{7}{*}{ \#Brexit } & degree & 0.0025 & 0.0029 & 0.0049 & 0.0199 & 0.0258 & 0.0390 & 0.1595 & 0.2487 & 0.2751 \\
\hline & closeness & 0.0122 & 0.0140 & 0.0241 & 0.0447 & 0.0566 & 0.0856 & 0.2669 & 0.3809 & 0.4213 \\
\hline & betweenness & 0.0144 & 0.0165 & 0.0284 & 0.0597 & 0.0745 & 0.1126 & 0.2823 & 0.3981 & 0.4403 \\
\hline & eigenvector & 0.015 & 0.0145 & 0.0296 & 0.0604 & 0.0743 & 0.1085 & 0.29 & 0.3965 & 0.4393 \\
\hline & page & 0.0138 & 0.0158 & 0.0271 & 0.0491 & 0.0620 & 0.0937 & 0.2720 & 0.3866 & 0.4277 \\
\hline & clustering & 0.0247 & 0.0279 & 0.0481 & 0.0377 & 0.0480 & 0.0726 & 0.2707 & 0.3852 & 0.4261 \\
\hline & kcore & 0.0034 & 0.0039 & 0.0038 & 0.0207 & 0.0269 & 0.0406 & 0.1608 & 0.2504 & 0.2771 \\
\hline \multirow{7}{*}{ \#covid } & degree & 0.3592 & 0.2189 & 0.5211 & 0.4639 & 0.302 & 0.6294 & 0.5951 & 0.4236 & 0.8212 \\
\hline & close & 0.361 & 0.2202 & 0.5211 & 0.401 & 0.2508 & 0.5664 & 0.4989 & 0.3324 & 0.662 \\
\hline & betwe & 0.3654 & 0.2235 & 0.5352 & 0.4675 & 0.3051 & 0.6294 & 0.5949 & 0.4234 & 0.8184 \\
\hline & eigen & 0.361 & 0.2202 & 0.5211 & 0.398 & 0.2484 & 0.5594 & 0.4995 & 0.3329 & 0.662 \\
\hline & pagerank & 0.3558 & 0.2164 & 0.5211 & 0.4694 & 0.3067 & 0.6434 & 0.5243 & 0.3553 & 0.6927 \\
\hline & ing & 0.5231 & 0.3542 & 0.7183 & 0.6055 & 0.4342 & 0.8531 & 0.6256 & 0.4552 & 0.8939 \\
\hline & kco & 0.3662 & 0.2124 & 0.5211 & 0.4642 & 0.2505 & 0.6445 & 0.6161 & 0.4142 & 0.6962 \\
\hline \multirow{7}{*}{ \#pandemi } & $\sigma_{0}$ & 0.3462 & 0.2093 & 0.5 & 0.3445 & 0.2081 & 0.5035 & 0.3563 & 0.2168 & 0.5196 \\
\hline & clos & 0.3429 & 0.2069 & 0.507 & 0.338 & 0.2034 & 0.5035 & 0.3486 & 0.2111 & 0.5112 \\
\hline & betweenness & 0.3429 & 0.2069 & 0.507 & 0.3462 & 0.2093 & 0.5035 & 0.3563 & 0.2168 & 0.5196 \\
\hline & eigenvector & 0.3429 & 0.2069 & 0.507 & 0.338 & 0.2034 & 0.5035 & 0.3486 & 0.2111 & 0.5112 \\
\hline & & 0.3429 & 0.2069 & 0.507 & 0.3429 & 0.2069 & 0.5035 & 0.3435 & 0.2073 & 0.5056 \\
\hline & clustering & 0.3663 & 0.2242 & 0.5211 & 0.3527 & 0.2141 & 0.5105 & 0.3563 & 0.2168 & 0.5196 \\
\hline & kcore & 0.3412 & 0.2003 & 0.5011 & 0.3132 & 0.2918 & 0.5535 & 0.3561 & 0.2504 & 0.5152 \\
\hline \multirow{7}{*}{ \#quarantine } & prats & 0.3302 & 0.1977 & 0.493 & 0.3318 & 0.1989 & 0.4965 & 0.3333 & 0.2 & 0.5 \\
\hline & & 0.3302 & 0.1977 & 0.493 & 3318 & 0.1989 & 0.4 & 0.3333 & 0. & 0.5 \\
\hline & betwe & 0.3302 & 0.1977 & 0.493 & 0.3318 & 0.1989 & 0.4965 & 0.3333 & 0. & 0.5 \\
\hline & eigen & 0.3302 & 0.1977 & 0.493 & 0.3318 & 0.1989 & 0.4965 & 0.3333 & 0.2 & 0.5 \\
\hline & & 0.3302 & 0.1977 & 0.493 & 0.3318 & 0.1989 & 0.4965 & 0.3333 & 0.2 & 0.5 \\
\hline & & 0.3302 & 0.1977 & 0.493 & 0.3318 & 0.1989 & 0.4965 & 0.3333 & 0. & 0.5 \\
\hline & & 0.3302 & 0.1977 & 0. & 18 & 0.1989 & & 0.3333 & 0.2 & 0.5 \\
\hline \multirow{7}{*}{ \#lockc } & & 0.3333 & 0.2 & 0.493 & 0.3412 & 0.2057 & 0.5035 & 0.3454 & 0.2087 & 0.5084 \\
\hline & clos & 0.3302 & 0.1977 & 0.493 & 0.338 & 0.2034 & 0.5035 & 0.3422 & 0.2064 & 0.5056 \\
\hline & betweenness & 0.3333 & 0.2 & 0.493 & 0.3541 & 0.2151 & 0.5175 & 0.346 & 0.2092 & 0.5084 \\
\hline & eigenvector & 0.3302 & 0.1977 & 0.493 & 0.3364 & 0.2022 & 0.5035 & 0.3422 & 0.2064 & 0.5056 \\
\hline & & 0.3333 & 0.2 & 0.493 & 0.3412 & 0.2057 & 0.50 & 0.3415 & 0.2059 & 0.5056 \\
\hline & ng & 0.3333 & 0.2 & 0.493 & 0.3541 & 0.2151 & 0.5175 & 0.3463 & 0.2094 & 0.5084 \\
\hline & kcore & 0.3302 & 0.1977 & 0.493 & 0.3114 & 0.2007 & 0.5113 & 0.3414 & 0.2112 & 0.5204 \\
\hline \multirow{7}{*}{ \#mask } & degree & 0.3302 & 0.1977 & 0.493 & 0.3318 & 0.1989 & 0.4965 & 0.345 & 0.2085 & 0.5084 \\
\hline & close & 0.3365 & 0.2023 & 0.493 & 0.3349 & 0.2011 & 0.4965 & 0.3358 & 0.2018 & 0.5 \\
\hline & betweenness & 0.3302 & 0.1977 & 0.493 & 0.3318 & 0.1989 & 0.4965 & 0.345 & 0.2085 & 0.5084 \\
\hline & & 0.3365 & 0.2023 & 0.493 & 0.3349 & 0.2011 & 0.4965 & 0.3358 & 0.2018 & 0.5 \\
\hline & pagerank & 0.3302 & 0.1977 & 0.493 & 0.3318 & 0.1989 & 0.4965 & 0.3447 & 0.2082 & 0.5084 \\
\hline & clustering & 0.3302 & 0.1977 & 0.493 & 0.3333 & 0.2 & 0.4965 & 0.345 & 0.2085 & 0.5084 \\
\hline & kcore & 0.3132 & 0.1878 & 0.493 & 0.3318 & 0.1887 & 0.4965 & 0.3321 & 0.2111 & 0.5 \\
\hline
\end{tabular}

It is interesting to note here that with an increase in the percentage of seed nodes used, the similarity between the actual seed nodes and the influential seed nodes using similarity measures also increases. This is because choosing a larger set as seed nodes increases the probability of overlap.

In Figure 3, similarity between actual and influential seeds, with respect to different seed node selection algorithms, in terms of the different similarity indexes for the keyword \#Brexit have been compared. The maximum number of influential nodes for \#Brexit is 4511 . It is to be noted 

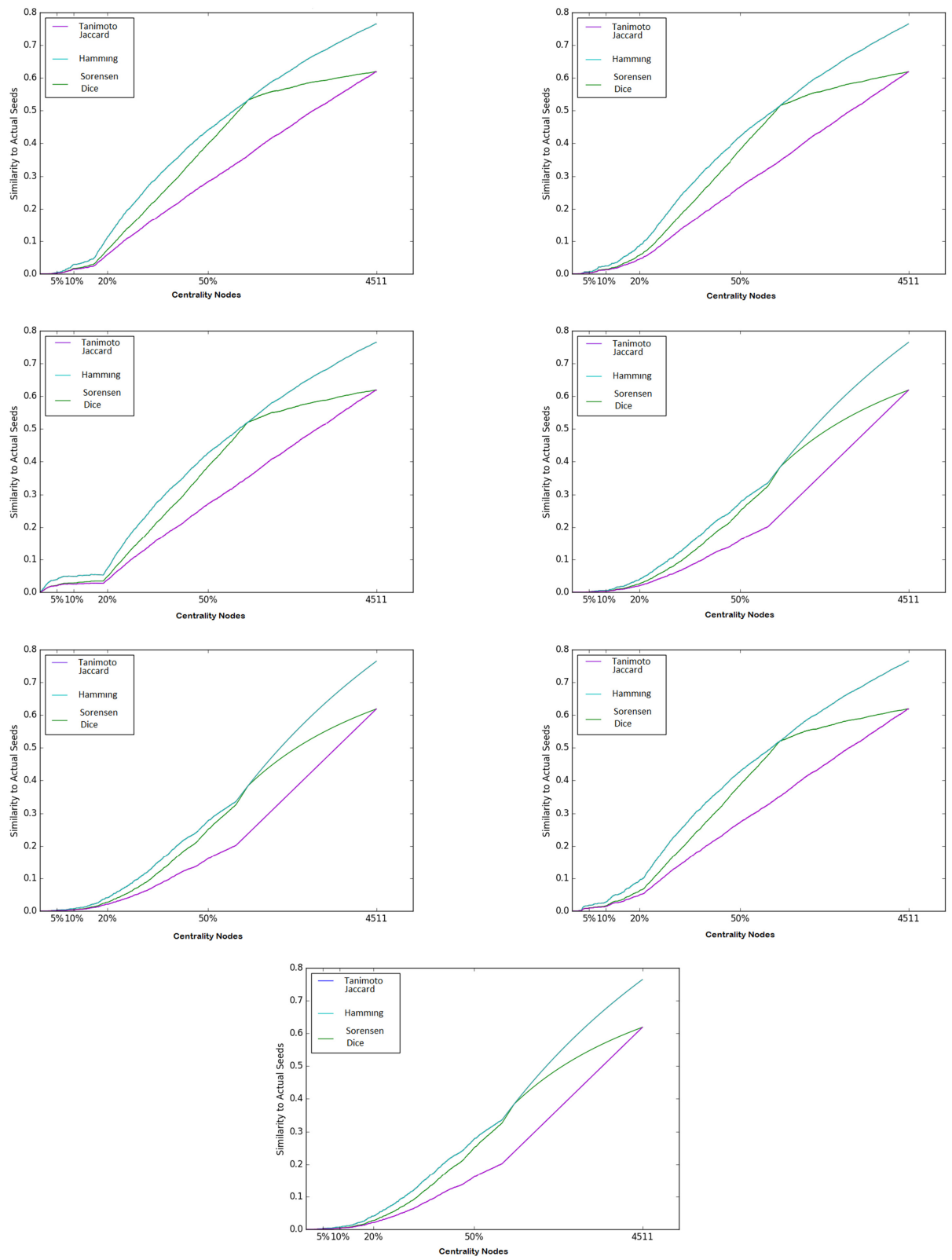

Fig. 3. Comparison of similarity between actual and influential seeds for different centrality measures for hashtags(\#) Brexit. Moving from left to right, beginning with the first row, the centrality algorithms depicted are betweenness, closeness, clustering, degree, eigenvector, pagerank, and K-core.

that in the chart, only Tanimoto, Dice, and Hamming curves are distinctively obtained. Jaccard and Sorensen curves coincide perfectly with Tanimoto and Dice, respectively. For all centrality measures, similarity indexes with actual seeds saturated in almost the same way at around $60 \%$ to $80 \%$. For smaller sets of data, Betweenness centrality is generally most similar to the actual seeds. 

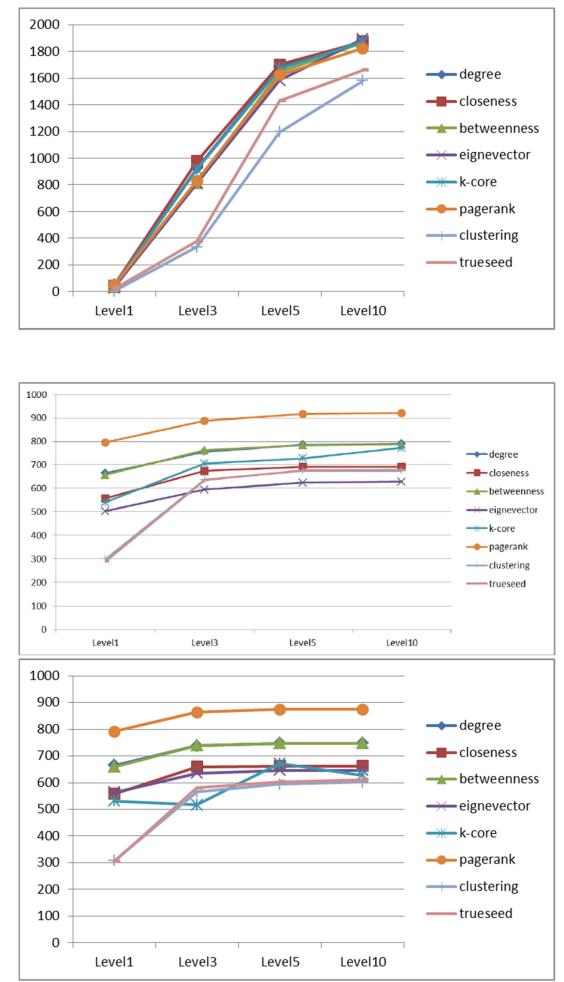

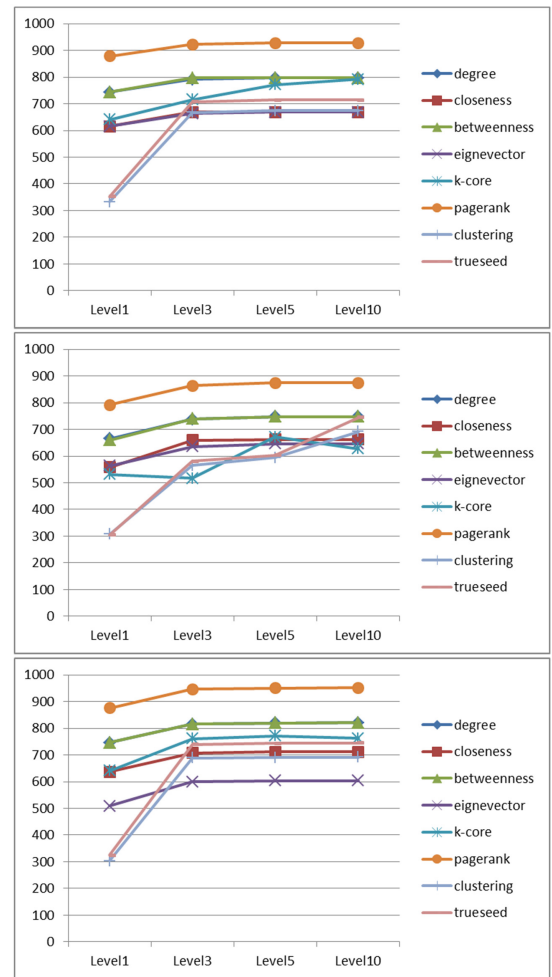

Fig. 4. Comparison of Performance of Centrality Measures with True Seeds for Breadth First Search Model with keywords \#Brexit, \#covid, \#pandemic, \#lockdown, \#quarantine, \#mask respectively moving from left to right, beginning with the first row

\subsection{Information Propagation}

In this section, the effect of simulating the spread of information from the highest centrality nodes, as discussed in the previous section, is shown. For studying the level of dissemination through centrality measures as seed nodes, the simulation is carried out using Breadth First Search, Random Walk, SIR, and Forest Fire.

4.3.1 Breadth First Search. In Table 5, a comparative analysis of information propagation from different centrality nodes along with an actual seed using Breadth First Search is shown for six datasets of \#Brexit, \#covid, \#pandemic, \#quarantine, \#lockdown, and \#mask. The columns list the number of affected nodes after 1, 3, 5, and 10 levels or steps of propagation.

From Table 5, it can be observed that PageRank, Betweenness, and Degree are the three frontrunners in maximizing information propagation. Closeness, K-core and Eigenvector centrality are also promising centrality measures for seed selection. All of these six methods are considerably better in seed selection compared to the true seed identified using the two-phase algorithm. Clustering coefficient is consistently a poor measure for seed selection, as can be seen from this table.

Figure 4 represents the performance of highest centrality measures with True Seeds as the source of propagation when propagation is simulated through Breadth First Search. It is the graphical representation of Table 5, where previous statements are represented through the compare charts for all six hashtags. 
Table 5. Comparative Analysis of Information Propagation from Highest Centrality Measures as Seed Nodes Using Breadth First Search Propagation

\begin{tabular}{|c|c|c|c|c|c|c|c|c|c|c|c|c|c|}
\hline \multirow{2}{*}{ Experiment } & \multirow{2}{*}{ Properties } & \multicolumn{3}{|c|}{ Level 1} & \multicolumn{3}{|c|}{ Level 3} & \multicolumn{3}{|c|}{ Level 5} & \multicolumn{3}{|c|}{ Level 10} \\
\hline & & C1 & C2 & C3 & C1 & $C 2$ & C3 & $C 1$ & C2 & C3 & $C 1$ & $C 2$ & C3 \\
\hline \multirow{8}{*}{ \#Brexit } & degree & 49 & 30 & 15 & 924 & 755 & 501 & 1684 & 1640 & 1444 & 1874 & 1872 & 1786 \\
\hline & clos & 45 & 28 & 14 & 978 & 854 & 573 & 1701 & 1668 & 1597 & 1874 & 1872 & 1871 \\
\hline & etwee & 35 & 22 & 12 & 815 & 666 & 458 & 1645 & 1583 & 1379 & 1869 & 1869 & 1743 \\
\hline & eigen & 31 & 23 & 13 & 813 & 668 & 500 & 1582 & 1602 & 1524 & 1892 & 1892 & 1732 \\
\hline & kcor & 41 & 29 & 15 & 916 & 768 & 508 & 1672 & 1642 & 1668 & 1872 & 1872 & 1802 \\
\hline & page & 48 & 28 & 14 & 830 & 595 & 429 & 1623 & 1313 & 1279 & 1824 & 1524 & 1679 \\
\hline & & 4 & 5 & 9 & 334 & 376 & 444 & 1196 & 1294 & 1368 & 1581 & 1674 & 1730 \\
\hline & tru & 22 & 20 & 12 & 380 & 450 & 550 & 1432 & 1322 & 1490 & 1661 & 1752 & 1782 \\
\hline \multirow{8}{*}{ \#covid } & deg & 744 & 629 & 518 & 791 & 676 & 559 & 797 & 682 & 565 & 797 & 682 & 565 \\
\hline & close & 615 & 355 & 200 & 669 & 399 & 224 & 669 & 399 & 224 & 669 & 399 & 224 \\
\hline & betwe & 743 & 629 & 507 & 797 & 683 & 559 & 797 & 683 & 559 & 797 & 683 & 559 \\
\hline & eigen & 616 & 248 & 154 & 663 & 274 & 157 & 669 & 280 & 157 & 669 & 280 & 157 \\
\hline & $\mathrm{kcc}$ & 641 & 629 & 615 & 716 & 668 & 608 & 772 & 742 & 768 & 792 & 772 & 702 \\
\hline & pag & 878 & 661 & 556 & 922 & 705 & 593 & 928 & 711 & 599 & 928 & 711 & 599 \\
\hline & cl & 333 & 147 & 78 & 668 & 409 & 281 & 674 & 415 & 286 & 674 & 415 & 286 \\
\hline & $\operatorname{tr}$ & 352 & 189 & 128 & 708 & 545 & 484 & 714 & 551 & 490 & 714 & 551 & 490 \\
\hline \multirow{8}{*}{ \#pandemic } & & 665 & 569 & 429 & 757 & 661 & 516 & 786 & 69 & 54 & 790 & 694 & 549 \\
\hline & clos & 558 & 246 & 156 & 674 & 344 & 242 & 691 & 361 & & 691 & 361 & 259 \\
\hline & betwe & 658 & 560 & 336 & 762 & 664 & 433 & 784 & 686 & 455 & 787 & 689 & 458 \\
\hline & gen & 503 & 176 & 93 & 595 & 245 & 143 & 624 & 274 & 168 & 628 & 278 & 172 \\
\hline & $\mathrm{kc}$ & 541 & 529 & 515 & 706 & 720 & 705 & 727 & 702 & 7 & 772 & 772 & 722 \\
\hline & & 795 & 584 & 485 & 887 & 673 & 566 & 916 & 702 & 5 & 920 & 706 & 599 \\
\hline & $\mathrm{hg}$ & 300 & 125 & 68 & 637 & 372 & 244 & 675 & 408 & 27 & 675 & 408 & 278 \\
\hline & & 289 & 157 & 110 & 636 & 497 & 448 & 677 & 536 & 48 & 678 & 537 & 488 \\
\hline \multirow{8}{*}{ \#quarantine } & & 703 & 596 & 505 & 759 & 652 & 555 & 780 & 67 & 5 & 781 & 674 & 577 \\
\hline & & 575 & 271 & 154 & 655 & 339 & 211 & 659 & 3 & & 659 & 343 & 214 \\
\hline & betw & 698 & 591 & 498 & 778 & 671 & 576 & 782 & 675 & 58 & 782 & 675 & 580 \\
\hline & et & 581 & 217 & 116 & 637 & 249 & 134 & 658 & 269 & & 659 & 270 & 134 \\
\hline & $\mathrm{kc}$ & 514 & 509 & 505 & 516 & 586 & 518 & 672 & 642 & 6 & 762 & 752 & 725 \\
\hline & & 834 & 631 & 552 & 888 & 684 & 598 & 909 & 705 & & 910 & 706 & 620 \\
\hline & & 287 & 115 & 63 & 577 & 354 & 265 & 620 & 397 & 308 & 659 & 436 & 347 \\
\hline & & 288 & 140 & 89 & 619 & 469 & 417 & 681 & 53 & & 687 & 537 & 485 \\
\hline \multirow{8}{*}{ \#lockdown } & & 665 & 557 & 417 & 738 & 630 & 485 & 748 & 64 & 495 & 748 & 640 & 495 \\
\hline & & 558 & 245 & 147 & 659 & 335 & 193 & 662 & 33 & & 662 & 338 & 196 \\
\hline & ess & 659 & 569 & 380 & 739 & 649 & 455 & 748 & 658 & 4 & 748 & 658 & 464 \\
\hline & 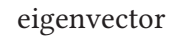 & 563 & 225 & 123 & 636 & 269 & 141 & 646 & 27 & & 646 & 279 & 148 \\
\hline & & 531 & 519 & 405 & 516 & 468 & 408 & 672 & 54 & 5 & 627 & 602 & 612 \\
\hline & & 79 & 598 & 474 & 864 & 670 & 538 & 874 & 68 & & 874 & 680 & 548 \\
\hline & & 307 & 137 & 68 & 564 & 251 & 127 & 594 & 2 & & 602 & 259 & 127 \\
\hline & & 304 & 156 & 105 & 581 & 429 & 378 & 604 & 452 & 401 & 612 & 460 & 409 \\
\hline \multirow{8}{*}{ \#mask } & & 746 & 647 & 509 & 816 & 717 & 577 & 820 & 721 & & 821 & 722 & 582 \\
\hline & & 637 & 332 & 189 & 707 & 387 & 226 & 711 & 391 & & 712 & 392 & 231 \\
\hline & & 746 & 645 & 469 & 816 & 715 & 537 & 820 & 719 & & 821 & 720 & 542 \\
\hline & 5,1 & 509 & 232 & 123 & 599 & 277 & 136 & 603 & 281 & 1 & 604 & 282 & 136 \\
\hline & & 641 & 529 & 551 & 761 & 768 & 708 & 772 & 752 & $7 \varepsilon$ & 762 & 772 & 712 \\
\hline & & 876 & 667 & 567 & 946 & 735 & 631 & 950 & 739 & 635 & 951 & 740 & 636 \\
\hline & & 303 & 138 & 73 & 686 & 465 & 334 & 690 & 469 & & 692 & 471 & 340 \\
\hline & trueseed & 324 & 171 & 120 & 739 & 581 & 529 & 743 & 585 & 533 & 745 & 587 & 535 \\
\hline
\end{tabular}

Figure 5 shows the comparison chart for \#Brexit, and the top five centrality values are selected and denoted as $\mathrm{C} 1, \mathrm{C} 2, \mathrm{C} 3, \mathrm{C} 4$, and $\mathrm{C} 5$, respectively. Thus, the graph for $\mathrm{C} 1$ traces propagation starting at the node with the highest centrality, whereas the graph for $\mathrm{C} 5$ traces the propagation starting at the node with the fifth highest centrality. From the chart, it is evident that for true seeds, it takes three to four hops to affect $10 \%$ of the total population. As mentioned earlier, total population for \#Brexit is 4511, and after six hops the information reaches its saturation level (i.e., almost 


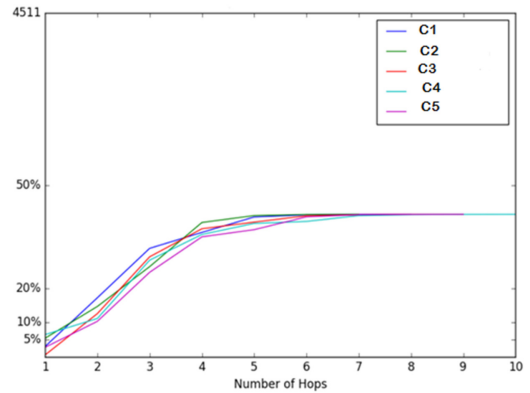

(A)

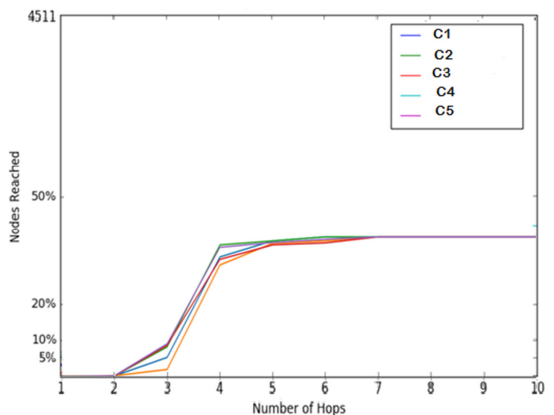

(B)

Fig. 5. Comparative graph of information propagation using Breadth First Search for hashtags(\#) Brexit with seeds displaying the highest K-core Centrality (a) with True Seed (b).

$50 \%$ of total data). But when the information is propagated from a seed with high betweenness centrality, it enjoys an initial head start. In Breadth First Search, one hop of all possible paths from the concerned node is checked for information propagation. Since a node with high betweenness centrality has a higher number of unique paths passing through it compared to other nodes, it therefore has higher chances of fanning out to a higher number of nodes in every step/hop. As such, $10 \%$ of the total population is affected after two hops, and the saturation point is reached within three hops.

4.3.2 Forest Fire. In Table 6, information propagation using Forest Fire for the same six datasets is represented. The columns list the number of affected nodes when considering the first (C1), second (C2), and third (C3) most favorable centrality measure, after 1, 3, 5, and 10 levels or steps of propagation. The experiments were conducted with the probability of new trees growing in the forest $(p)$ set at 0.01 and the probability of a tree catching on fire $(f)$ set at 0.01 .

From Table 6, it is observed that PageRank, Betweenness, Eigenvector, K-core, Closeness, and Node degree centrality are all good measures for influential seed nodes. Contrary to the other propagation models, in forest fire, the performance of clustering coefficient is also considerably improved. True seed predicted using the two-phase algorithm is outperformed by all centrality measures in almost all datasets. Another important observation is that all influential seeds reach their saturation values of approximately $48 \%$ at almost the same five-hop distance.

Figure 6 represents the performance of highest centrality measures with True Seeds as the source of propagation when propagation is simulated through Forest Fire. From the figures, it can be observed that centrality measures have better performance in terms of affected nodes in comparison to true seed.

Figure 7 reflects the level of propagation for \#Brexit when seed nodes are determined by the highest Closeness centrality and clustering coefficient. From the figure, it is evident that when information starts propagating the seeds derived by clustering coefficient, it reaches merely $5 \%$ of the total population, irrespective of the influential seeds-that is, it shows the same trend for all five seed nodes. Using closeness centrality as the seed node selection, $15 \%$ to $20 \%$ of the total population is reach in the second hop. For C1, more than $50 \%$ of the population is reached at saturation.

4.3.3 Random Walk. Table 7 provides the data as Table 6, corresponding to the user of the Random Walk algorithm for propagation. Hence, the structure is semantically identical. Results are tabulated at levels $10,30,50$, and 100 for the low propagation in the network.

From Table 7, it is also evident that the saturation levels for propagation of all influential seed nodes except clustering coefficient and the original seed node are identical. The poor performance 
Table 6. Comparative Analysis of Information Propagation from Different Seed Nodes Using Forest Fire Propagation

\begin{tabular}{|c|c|c|c|c|c|c|c|c|c|c|c|c|c|}
\hline \multirow{2}{*}{ Experiment } & \multirow{2}{*}{ Properties } & \multicolumn{3}{|c|}{ Level 1} & \multicolumn{3}{|c|}{ Level 3} & \multicolumn{3}{|c|}{ Level 5} & \multicolumn{3}{|c|}{ Level 10} \\
\hline & & $C 1$ & $C 2$ & C3 & $C 1$ & $C 2$ & C3 & $C 1$ & $C 2$ & C3 & $C 1$ & $C 2$ & C3 \\
\hline \multirow{8}{*}{ \#Brexit } & degree & 560 & 337 & 282 & 1879 & 1400 & 1328 & 2203 & 1926 & 1996 & 2305 & 2130 & 2095 \\
\hline & closeness & 557 & 279 & 179 & 1899 & 1746 & 1598 & 2216 & 2024 & 1983 & 2324 & 2126 & 2086 \\
\hline & betweenness & 182 & 285 & 66 & 1556 & 1340 & 1462 & 1996 & 1995 & 1966 & 2122 & 2093 & 2126 \\
\hline & eigenvector & 162 & 193 & 184 & 1135 & 1616 & 1516 & 1614 & 2260 & 2520 & 2416 & 2448 & 2496 \\
\hline & kcore & 181 & 161 & 126 & 1549 & 1241 & 1376 & 1983 & 1937 & 2005 & 2104 & 2162 & 2128 \\
\hline & pagerank & 561 & 200 & 184 & 1898 & 1220 & 1553 & 2224 & 2001 & 2000 & 2317 & 2105 & 2111 \\
\hline & clustering & 43 & 49 & 47 & 717 & 620 & 540 & 1817 & 1846 & 1761 & 2107 & 2144 & 2098 \\
\hline & trueseed & 201 & 182 & 220 & 890 & 980 & 1123 & 1818 & 1834 & 1702 & 1889 & 1786 & 1890 \\
\hline \multirow{8}{*}{ \#covid } & degree & 15 & 17 & 19 & 134 & 180 & 100 & 249 & 263 & 218 & 477 & 503 & 452 \\
\hline & closeness & 18 & 16 & 9 & 66 & 121 & 158 & 199 & 221 & 275 & 498 & 480 & 471 \\
\hline & betweenness & 15 & 15 & 15 & 105 & 100 & 78 & 288 & 276 & 255 & 413 & 468 & 573 \\
\hline & eigenvector & 18 & 19 & 16 & 156 & 66 & 135 & 250 & 260 & 164 & 496 & 448 & 416 \\
\hline & kcore & 16 & 18 & 16 & 145 & 101 & 130 & 233 & 255 & 217 & 471 & 457 & 422 \\
\hline & pagerank & 20 & 13 & 13 & 97 & 92 & 137 & 271 & 228 & 215 & 439 & 519 & 520 \\
\hline & clustering & 22 & 19 & 22 & 84 & 100 & 162 & 275 & 219 & 205 & 514 & 468 & 538 \\
\hline & trueseed & 15 & 18 & 31 & 116 & 118 & 201 & 251 & 213 & 246 & 472 & 464 & 455 \\
\hline \multirow{8}{*}{ \#pandemic } & degree & 16 & 19 & 14 & 99 & 98 & 52 & 219 & 282 & 252 & 523 & 521 & 481 \\
\hline & closeness & 14 & 11 & 18 & 102 & 102 & 96 & 284 & 257 & 271 & 521 & 478 & 493 \\
\hline & betweenness & 12 & 17 & 18 & 109 & 108 & 78 & 219 & 242 & 244 & 459 & 505 & 461 \\
\hline & eigenvector & 20 & 17 & 24 & 113 & 98 & 73 & 224 & 320 & 237 & 504 & 529 & 489 \\
\hline & kcore & 14 & 16 & 18 & 101 & 100 & 101 & 231 & 220 & 217 & 501 & 504 & 480 \\
\hline & pagerank & 15 & 17 & 19 & 101 & 109 & 141 & 279 & 207 & 248 & 458 & 514 & 511 \\
\hline & clustering & 18 & 22 & 15 & 130 & 103 & 129 & 221 & 275 & 229 & 518 & 524 & 479 \\
\hline & trueseed & 14 & 18 & 18 & 98 & 105 & 96 & 256 & 232 & 266 & 453 & 549 & 521 \\
\hline \multirow{8}{*}{ \#quarantine } & degree & 21 & 13 & 21 & 182 & 139 & 132 & 340 & 304 & 237 & 527 & 555 & 489 \\
\hline & closeness & 23 & 17 & 21 & 116 & 209 & 203 & 236 & 293 & 300 & 503 & 476 & 510 \\
\hline & betweenness & 18 & 17 & 14 & 124 & 183 & 86 & 308 & 279 & 244 & 533 & 525 & 540 \\
\hline & eigenvector & 16 & 17 & 14 & 179 & 137 & 114 & 279 & 342 & 261 & 513 & 558 & 539 \\
\hline & kcore & 15 & 17 & 14 & 149 & 169 & 98 & 277 & 287 & 261 & 502 & 500 & 504 \\
\hline & pagerank & 13 & 18 & 24 & 152 & 140 & 171 & 335 & 296 & 295 & 561 & 503 & 453 \\
\hline & clustering & 13 & 17 & 20 & 101 & 163 & 105 & 295 & 257 & 372 & 569 & 514 & 462 \\
\hline & trueseed & 13 & 14 & 14 & 110 & 120 & 143 & 379 & 267 & 323 & 509 & 524 & 487 \\
\hline \multirow{8}{*}{ \#lockdown } & degree & 16 & 13 & 17 & 141 & 157 & 175 & 296 & 294 & 275 & 469 & 486 & 449 \\
\hline & closeness & 15 & 19 & 16 & 128 & 73 & 186 & 293 & 239 & 282 & 467 & 499 & 464 \\
\hline & betweenness & 14 & 19 & 14 & 54 & 110 & 91 & 213 & 279 & 268 & 538 & 436 & 527 \\
\hline & eigenvector & 20 & 11 & 15 & 95 & 164 & 124 & 221 & 260 & 288 & 430 & 481 & 483 \\
\hline & kcore & 16 & 13 & 16 & 98 & 110 & 99 & 245 & 249 & 230 & 450 & 463 & 456 \\
\hline & pagerank & 11 & 13 & 13 & 99 & 155 & 93 & 238 & 236 & 234 & 510 & 458 & 459 \\
\hline & clustering & 15 & 6 & 18 & 157 & 143 & 191 & 256 & 251 & 344 & 460 & 480 & 394 \\
\hline & trueseed & 12 & 19 & 10 & 98 & 138 & 171 & 243 & 287 & 266 & 339 & 419 & 501 \\
\hline \multirow{8}{*}{ \#mask } & degree & 23 & 12 & 13 & 138 & 77 & 143 & 308 & 331 & 341 & 496 & 521 & 512 \\
\hline & closeness & 18 & 15 & 15 & 170 & 161 & 99 & 236 & 253 & 321 & 521 & 536 & 530 \\
\hline & betweenness & 14 & 16 & 18 & 89 & 94 & 119 & 247 & 268 & 234 & 541 & 518 & 498 \\
\hline & eigenvector & 14 & 22 & 11 & 121 & 116 & 157 & 287 & 314 & 242 & 472 & 545 & 577 \\
\hline & kcore & 18 & 20 & 14 & 110 & 108 & 124 & 265 & 301 & 241 & 451 & 519 & 499 \\
\hline & pagerank & 13 & 16 & 11 & 136 & 113 & 106 & 283 & 239 & 306 & 524 & 499 & 474 \\
\hline & clustering & 23 & 14 & 26 & 155 & 108 & 102 & 215 & 312 & 242 & 552 & 564 & 472 \\
\hline & trueseed & 12 & 21 & 10 & 114 & 115 & 113 & 253 & 286 & 296 & 459 & 503 & 512 \\
\hline
\end{tabular}

of clustering coefficient may be attributed to the fact that it confines itself to a local community within the network.

Figure 8 represents the performance of centrality measures with True Seeds as the source of propagation when propagation is simulated through Random Walk. Except \#Brexit, for all other 

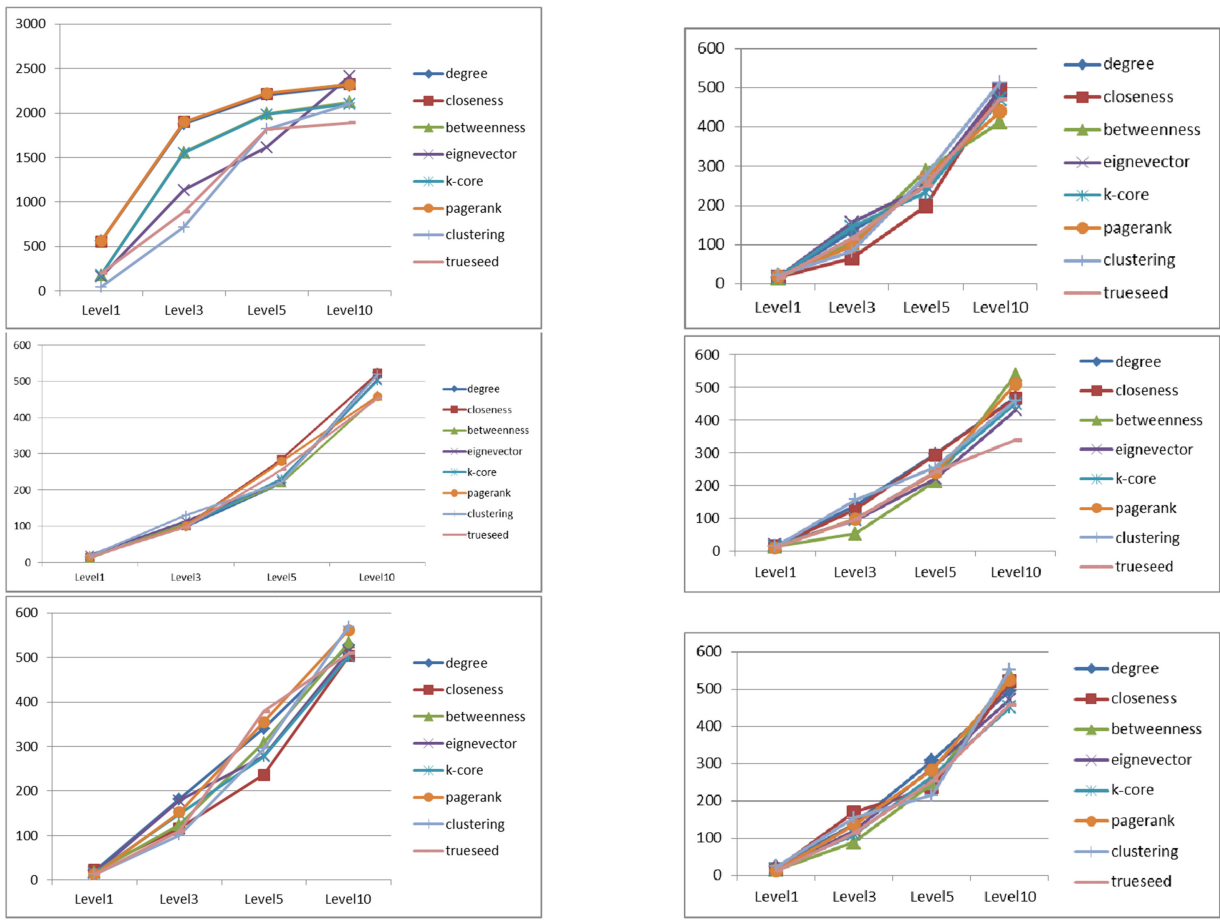

Fig. 6. Comparison of performance of centrality measures with true seeds for the Forest Fire model with keywords \#Brexit, \#covid, \#pandemic, \#lockdown, \#quarantine, and \#mask respectively from top left to right, beginning with the first row.
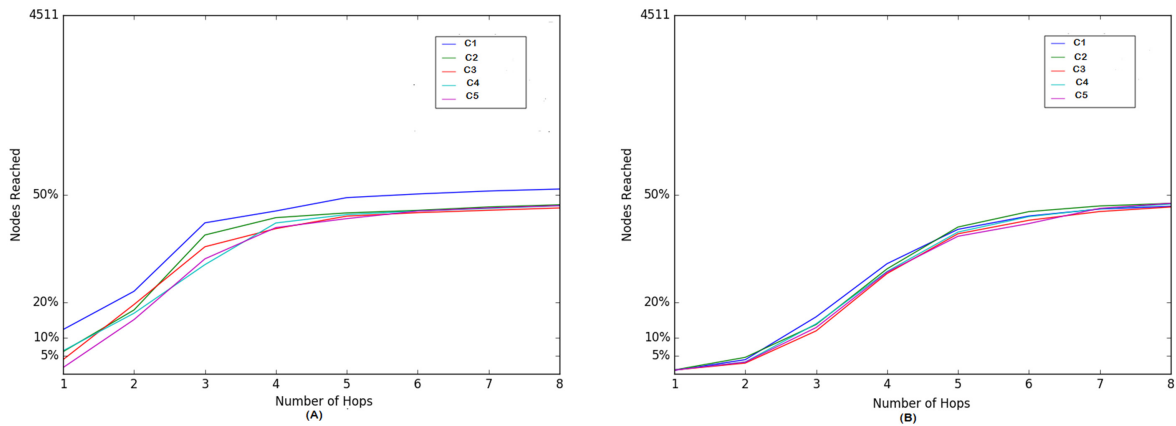

Fig. 7. Comparative graph of information propagation using Forest Fire propagation from seed nodes for hashtags(\#) Brexit highest Closeness centrality (a) and highest Clustering coefficient (b).

\#, as compared to original seed, all centrality measures show better influence. For \#Brexit as well, only clustering coefficient fails to achieve better performance.

In Figure 9, the extent of propagation for \#Brexit is shown for the degree and PageRank seed selection strategies. Degree outperforms PageRank during the initial stages of propagation. Its results are dependent on the particular seed node selected, whereas the influence of PageRank is almost identical for the top five centrality measures.

From Figure 9, it is evident that propagation from all influential seeds are overlapping in nature, especially for PageRank centrality. The saturation level is only $20 \%$ to the total population of 
Table 7. Comparative Analysis of Information Propagation from Different Seed Nodes Using Random Walk

\begin{tabular}{|c|c|c|c|c|c|c|c|c|c|c|c|c|c|}
\hline \multirow{2}{*}{ Experiment } & \multirow{2}{*}{ Properties } & \multicolumn{3}{|c|}{ Level 10} & \multicolumn{3}{|c|}{ Level 30} & \multicolumn{3}{|c|}{ Level 50} & \multicolumn{3}{|c|}{ Level 100} \\
\hline & & $C 1$ & $C 2$ & C3 & $C 1$ & C2 & C3 & C1 & $C 2$ & C3 & C1 & C2 & C3 \\
\hline \multirow{8}{*}{ \#Brexit } & degree & 560 & 337 & 282 & 1879 & 1400 & 1328 & 2203 & 1926 & 1996 & 2305 & 2130 & 2095 \\
\hline & closeness & 557 & 279 & 179 & 1899 & 1746 & 1598 & 2216 & 2024 & 1983 & 2324 & 2126 & 2086 \\
\hline & betweenness & 182 & 285 & 66 & 1556 & 1340 & 1462 & 1996 & 1995 & 1966 & 2122 & 2093 & 2126 \\
\hline & eigenvector & 181 & 280 & 156 & 1796 & 1741 & 1489 & 2100 & 1989 & 2001 & 2254 & 2080 & 2127 \\
\hline & kcore & 453 & 410 & 460 & 965 & 962 & 981 & 1165 & 1150 & 1167 & 1357 & 1350 & 1355 \\
\hline & pagerank & 561 & 200 & 184 & 1898 & 1220 & 1553 & 2224 & 2001 & 2000 & 2317 & 2105 & 2111 \\
\hline & clustering & 43 & 49 & 47 & 717 & 620 & 540 & 1817 & 1846 & 1761 & 2107 & 2144 & 2098 \\
\hline & true & 161 & 242 & 82 & 1230 & 1242 & 1122 & 1740 & 1732 & 1712 & 1901 & 1911 & 1991 \\
\hline \multirow{8}{*}{ \#covid } & degree & 1114 & 436 & 210 & 1222 & 533 & 280 & 1251 & 576 & 307 & 1280 & 605 & 338 \\
\hline & close & 1157 & 501 & 274 & 1256 & 538 & 283 & 1294 & 561 & 286 & 1325 & 580 & 296 \\
\hline & betweenness & 1113 & 435 & 209 & 1209 & 529 & 275 & 1247 & 570 & 310 & 1279 & 600 & 337 \\
\hline & eigenvector & 1158 & 501 & 279 & 1251 & 531 & 281 & 1293 & 542 & 281 & 1325 & 554 & 281 \\
\hline & kcore & 1111 & 410 & 234 & 1212 & 531 & 276 & 1242 & 553 & 290 & 1299 & 536 & 280 \\
\hline & page & 1080 & 430 & 210 & 1161 & 513 & 275 & 1202 & 559 & 310 & 1233 & 587 & 336 \\
\hline & clust & 1104 & 433 & 215 & 1157 & 454 & 230 & 1171 & 461 & 236 & 1191 & 468 & 238 \\
\hline & trues & 858 & 373 & 216 & 904 & 401 & 230 & 920 & 403 & 237 & 935 & 414 & 240 \\
\hline \multirow{8}{*}{ \#pandemic } & & 1044 & 411 & 196 & 1180 & 540 & 262 & 1234 & 587 & 303 & 1271 & 626 & 344 \\
\hline & closeness & 1084 & 452 & 202 & 1220 & 506 & 232 & 1264 & 534 & 248 & 1308 & 556 & 260 \\
\hline & betweenness & 1046 & 414 & 204 & 1179 & 535 & 274 & 1224 & 587 & 303 & 1270 & 629 & 341 \\
\hline & eigenvector & 1073 & 456 & 222 & 1188 & 505 & 246 & 1228 & 526 & 259 & 1276 & 542 & 268 \\
\hline & kcore & 1056 & 412 & 203 & 1124 & 522 & 232 & 1232 & 567 & 261 & 1250 & 535 & 235 \\
\hline & pag & 1021 & 409 & 187 & 1135 & 516 & 237 & 1176 & 559 & 282 & 1224 & 605 & 311 \\
\hline & clust & 1032 & 408 & 203 & 1101 & 446 & 219 & 1130 & 461 & 230 & 1159 & 471 & 239 \\
\hline & $\mathrm{d}$ & 741 & 328 & 188 & 787 & 350 & 203 & 807 & 356 & 208 & 824 & 361 & 210 \\
\hline \multirow{8}{*}{ \#lockdown } & deg & 1005 & 414 & 205 & 1167 & 558 & 297 & 1227 & 615 & 341 & 1294 & 679 & 387 \\
\hline & close & 1015 & 431 & 242 & 1179 & 507 & 279 & 1252 & 546 & 291 & 1303 & 582 & 299 \\
\hline & betweenness & 1010 & 428 & 206 & 1185 & 583 & 300 & 1238 & 650 & 333 & 1298 & 705 & 370 \\
\hline & eigenvector & 1010 & 428 & 243 & 1175 & 495 & 279 & 1229 & 527 & 294 & 1289 & 560 & 305 \\
\hline & $\mathrm{kco}$ & 1002 & 421 & 220 & 1165 & 562 & 281 & 1225 & 550 & 267 & 1257 & 550 & 355 \\
\hline & $\mathrm{k}$ & 989 & 407 & 205 & 1113 & 521 & 282 & 1167 & 583 & 328 & 1209 & 629 & 366 \\
\hline & $n g$ & 969 & 373 & 189 & 1053 & 408 & 209 & 1086 & 429 & 222 & 1102 & 438 & 225 \\
\hline & & 723 & 315 & 178 & 784 & 344 & 195 & 793 & 352 & 204 & 819 & 360 & 211 \\
\hline \multirow{8}{*}{ \#quarantine } & deg & 1090 & 421 & 200 & 1192 & 528 & 263 & 1223 & 548 & 300 & 1261 & 587 & 324 \\
\hline & close & 1139 & 491 & 263 & 1232 & 516 & 275 & 1273 & 528 & 283 & 1302 & 541 & 292 \\
\hline & betweenness & 1090 & 421 & 200 & 1185 & 510 & 264 & 1226 & 556 & 298 & 1248 & 579 & 318 \\
\hline & eigenvector & 1137 & 498 & 269 & 1220 & 514 & 272 & 1269 & 527 & 273 & 1299 & 531 & 273 \\
\hline & kcore & 1099 & 441 & 260 & 1155 & 511 & 241 & 1234 & 554 & 276 & 1251 & 510 & 285 \\
\hline & $n k$ & 1029 & 412 & 200 & 1116 & 486 & 258 & 1146 & 525 & 286 & 1182 & 556 & 311 \\
\hline & clust & 1071 & 425 & 206 & 1120 & 453 & 223 & 1139 & 460 & 233 & 1151 & 466 & 234 \\
\hline & & 755 & 328 & 183 & 788 & 343 & 192 & 797 & 348 & 194 & 810 & 353 & 196 \\
\hline \multirow{8}{*}{ \#mask } & degree & 1073 & 431 & 202 & 1183 & 557 & 270 & 1236 & 575 & 306 & 1268 & 618 & 328 \\
\hline & closeness & 1134 & 483 & 266 & 1248 & 531 & 279 & 1293 & 550 & 284 & 1325 & 561 & 287 \\
\hline & betweenness & 1074 & 430 & 217 & 1188 & 538 & 288 & 1234 & 592 & 328 & 1269 & 621 & 359 \\
\hline & eigenvector & 1127 & 492 & 262 & 1223 & 524 & 270 & 1256 & 539 & 273 & 1289 & 553 & 275 \\
\hline & kcore & 1072 & 430 & 241 & 1195 & 542 & 270 & 1236 & 550 & 257 & 1271 & 557 & 257 \\
\hline & pagerank & 1051 & 422 & 202 & 1165 & 525 & 265 & 1203 & 569 & 297 & 1222 & 598 & 325 \\
\hline & clustering & 1059 & 407 & 203 & 1119 & 433 & 213 & 1137 & 444 & 218 & 1159 & 453 & 221 \\
\hline & trueseed & 787 & 344 & 203 & 832 & 370 & 215 & 852 & 374 & 221 & 864 & 383 & 223 \\
\hline
\end{tabular}

the network. For the simulation of propagation, Random Walk is a poor choice for a large-scale network due to the problem that slow mixing in graphs consists of a large number of communities (or mostly in networks where community structure is dominating). In such scenarios, random walk needs a large number of samples and a long period to reach the steady-state with the desired 

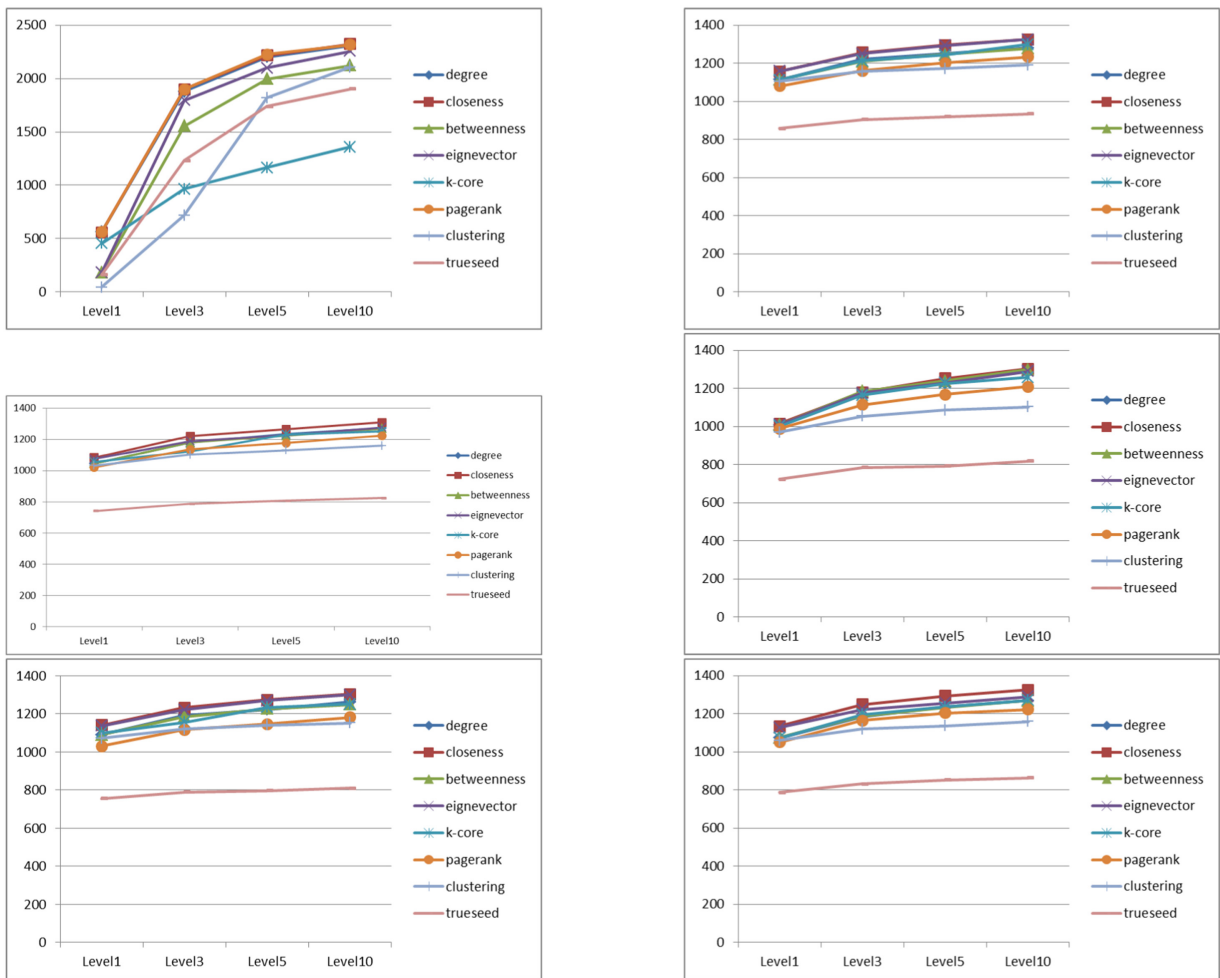

Fig. 8. Comparison of performance of centrality measures with true seeds for the Random Walk ,odel with keywords \#Brexit, \#covid, \#pandemic, \#lockdown, \#quarantine, and \#mask respectively from top left to right, beginning with the first row.
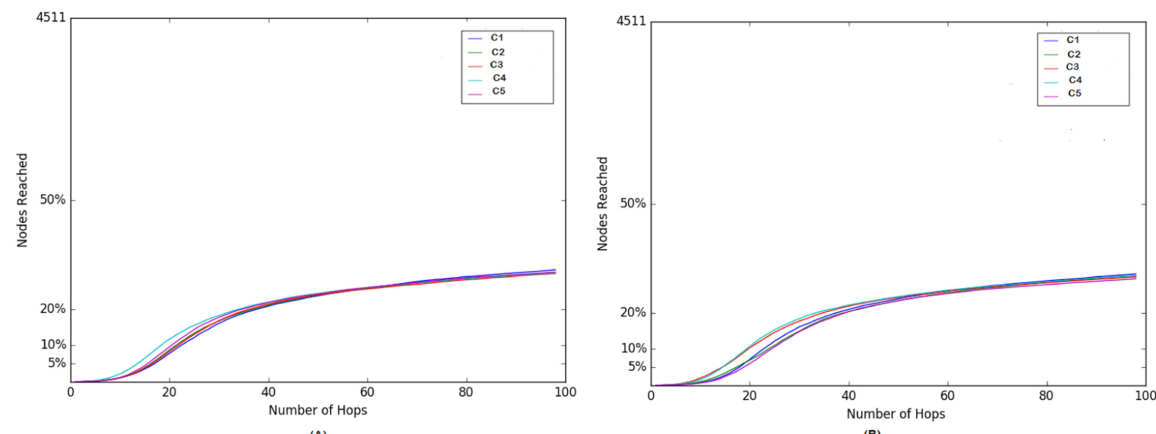

Fig. 9. Comparative graph of information propagation using Random Walk propagation from seed nodes for hashtags(\#) Brexit highest Degree (a) and highest PageRank (b).

accuracy. Moreover, it tends to be limited within a close community structure and cannot spread widely.

4.3.4 SIR Model. Table 8 provides the data corresponding to the SIR model for propagation. Here, the seed nodes, either chosen using the centrality measures or the true seeds chosen using 
Table 8. Comparative Analysis of Information Propagation from Different Seed Nodes Using the SIR Model

\begin{tabular}{|c|c|c|c|c|c|c|c|c|c|c|c|c|c|}
\hline \multirow{2}{*}{ Experiment } & \multirow{2}{*}{ Properties } & \multicolumn{3}{|c|}{ Level 1} & \multicolumn{3}{|c|}{ Level 3} & \multicolumn{3}{|c|}{ Level 5} & \multicolumn{3}{|c|}{ Level 10} \\
\hline & & $C 1$ & $C 2$ & C3 & $C 1$ & $C 2$ & C3 & $C 1$ & C2 & C3 & C1 & $C 2$ & C3 \\
\hline \multirow{8}{*}{ \#Brexit } & degree & 580 & 339 & 262 & 1697 & 1410 & 1526 & 2005 & 1728 & 1778 & 2503 & 2150 & 2073 \\
\hline & closeness & 559 & 297 & 197 & 1677 & 1948 & 1576 & 2218 & 2121 & 1763 & 2520 & 2128 & 2068 \\
\hline & betweenness & 162 & 278 & 156 & 1736 & 1430 & 1264 & 1966 & 1559 & 1696 & 2121 & 2093 & 2112 \\
\hline & eigenvector & 161 & 260 & 158 & 1978 & 1941 & 1467 & 2001 & 1767 & 2100 & 2232 & 2060 & 2129 \\
\hline & kcore & 451 & 429 & 415 & 1545 & 1550 & 1542 & 1962 & 1950 & 1972 & 2310 & 2300 & 2302 \\
\hline & pagerank & 341 & 255 & 164 & 1696 & 1440 & 1335 & 2442 & 2110 & 2101 & 2173 & 2051 & 2110 \\
\hline & clustering & 45 & 47 & 49 & 919 & 820 & 560 & 1619 & 1648 & 1981 & 2109 & 2166 & 2076 \\
\hline & trueseed & 121 & 242 & 130 & 1200 & 1142 & 1222 & 1701 & 1702 & 1712 & 1901 & 1901 & 1992 \\
\hline \multirow{8}{*}{ \#covid } & degree & 722 & 322 & 167 & 722 & 360 & 191 & 722 & 399 & 222 & 722 & 520 & 315 \\
\hline & closeness & 722 & 322 & 167 & 722 & 362 & 193 & 722 & 402 & 225 & 722 & 507 & 313 \\
\hline & betweenness & 722 & 322 & 166 & 722 & 362 & 193 & 722 & 399 & 224 & 722 & 515 & 308 \\
\hline & eigenvector & 722 & 323 & 167 & 722 & 361 & 194 & 722 & 398 & 224 & 722 & 512 & 302 \\
\hline & kcore & 722 & 321 & 166 & 722 & 360 & 192 & 722 & 396 & 223 & 722 & 509 & 306 \\
\hline & pagerank & 722 & 323 & 167 & 722 & 360 & 193 & 722 & 402 & 223 & 722 & 513 & 309 \\
\hline & clustering & 722 & 321 & 167 & 722 & 360 & 193 & 722 & 400 & 221 & 722 & 496 & 311 \\
\hline & trueseed & 580 & 252 & 129 & 604 & 287 & 151 & 631 & 320 & 177 & 694 & 436 & 256 \\
\hline \multirow{8}{*}{ \#pandemic } & degree & 681 & 305 & 157 & 681 & 341 & 180 & 681 & 381 & 208 & 681 & 483 & 298 \\
\hline & closeness & 681 & 305 & 157 & 681 & 339 & 182 & 681 & 381 & 211 & 681 & 484 & 290 \\
\hline & betweenness & 681 & 303 & 156 & 681 & 340 & 182 & 681 & 378 & 209 & 681 & 479 & 300 \\
\hline & eigenvector & 681 & 305 & 157 & 681 & 338 & 182 & 681 & 375 & 211 & 681 & 481 & 297 \\
\hline & kcore & 681 & 301 & 155 & 681 & 337 & 180 & 681 & 375 & 208 & 681 & 476 & 297 \\
\hline & pagerank & 681 & 305 & 157 & 681 & 339 & 181 & 681 & 380 & 210 & 681 & 483 & 293 \\
\hline & clustering & 681 & 305 & 157 & 681 & 342 & 181 & 681 & 381 & 209 & 681 & 476 & 296 \\
\hline & trueseed & 529 & 228 & 116 & 564 & 260 & 135 & 595 & 296 & 158 & 680 & 393 & 229 \\
\hline \multirow{8}{*}{ \#lockdown } & degree & 647 & 288 & 150 & 647 & 322 & 174 & 647 & 359 & 199 & 647 & 445 & 280 \\
\hline & closeness & 647 & 289 & 150 & 647 & 321 & 171 & 647 & 359 & 199 & 647 & 458 & 284 \\
\hline & betweenness & 647 & 289 & 150 & 647 & 322 & 173 & 647 & 360 & 200 & 647 & 454 & 282 \\
\hline & eigenvector & 647 & 289 & 150 & 647 & 322 & 171 & 647 & 356 & 199 & 647 & 453 & 279 \\
\hline & kcore & 647 & 287 & 150 & 647 & 320 & 170 & 647 & 354 & 200 & 647 & 456 & 279 \\
\hline & pagerank & 647 & 288 & 150 & 647 & 323 & 172 & 647 & 358 & 199 & 647 & 452 & 279 \\
\hline & clustering & 647 & 289 & 150 & 647 & 322 & 174 & 647 & 359 & 198 & 647 & 463 & 283 \\
\hline & trueseed & 522 & 227 & 116 & 552 & 259 & 135 & 582 & 293 & 158 & 650 & 386 & 227 \\
\hline \multirow{8}{*}{ \#quarantine } & degree & 685 & 306 & 158 & 685 & 343 & 181 & 685 & 377 & 210 & 685 & 482 & 288 \\
\hline & closeness & 685 & 306 & 157 & 685 & 344 & 183 & 685 & 382 & 208 & 685 & 492 & 297 \\
\hline & betweenness & 685 & 304 & 158 & 685 & 342 & 182 & 685 & 379 & 210 & 685 & 485 & 299 \\
\hline & eigenvector & 685 & 306 & 158 & 685 & 344 & 183 & 685 & 377 & 211 & 685 & 480 & 293 \\
\hline & kcore & 685 & 304 & 158 & 685 & 341 & 180 & 685 & 383 & 212 & 685 & 480 & 297 \\
\hline & pagerank & 685 & 307 & 158 & 685 & 343 & 182 & 685 & 378 & 212 & 685 & 494 & 291 \\
\hline & clustering & 685 & 307 & 158 & 685 & 343 & 183 & 685 & 380 & 210 & 685 & 485 & 295 \\
\hline & trueseed & 535 & 229 & 117 & 566 & 259 & 138 & 604 & 296 & 161 & 687 & 402 & 232 \\
\hline \multirow{8}{*}{ \#mask } & degree & 697 & 311 & 162 & 697 & 347 & 186 & 697 & 378 & 212 & 697 & 489 & 298 \\
\hline & closeness & 697 & 310 & 162 & 697 & 342 & 186 & 697 & 387 & 214 & 697 & 500 & 297 \\
\hline & betweenness & 697 & 309 & 162 & 697 & 344 & 185 & 697 & 385 & 215 & 697 & 493 & 295 \\
\hline & eigenvector & 697 & 310 & 162 & 697 & 347 & 187 & 697 & 381 & 216 & 697 & 491 & 298 \\
\hline & kcore & 697 & 310 & 162 & 697 & 347 & 185 & 697 & 381 & 214 & 697 & 490 & 296 \\
\hline & pagerank & 697 & 311 & 162 & 697 & 343 & 187 & 697 & 383 & 213 & 697 & 489 & 299 \\
\hline & clustering & 697 & 310 & 162 & 697 & 347 & 186 & 697 & 384 & 217 & 697 & 493 & 299 \\
\hline & trueseeds & 544 & 235 & 120 & 572 & 264 & 140 & 596 & 302 & 166 & 660 & 410 & 241 \\
\hline
\end{tabular}

the two-phase algorithm, are initially taken to be the infected nodes. All other nodes in the network are considered to be susceptible in the beginning, and no node is in the recovered state. Results are tabulated at levels $1,3,5$, and 10 . The probability of infection $(\beta)$ is set at 0.2 , and the proportion of infected persons recovering every unit time $(\lambda)$ is set at 0.1 . 

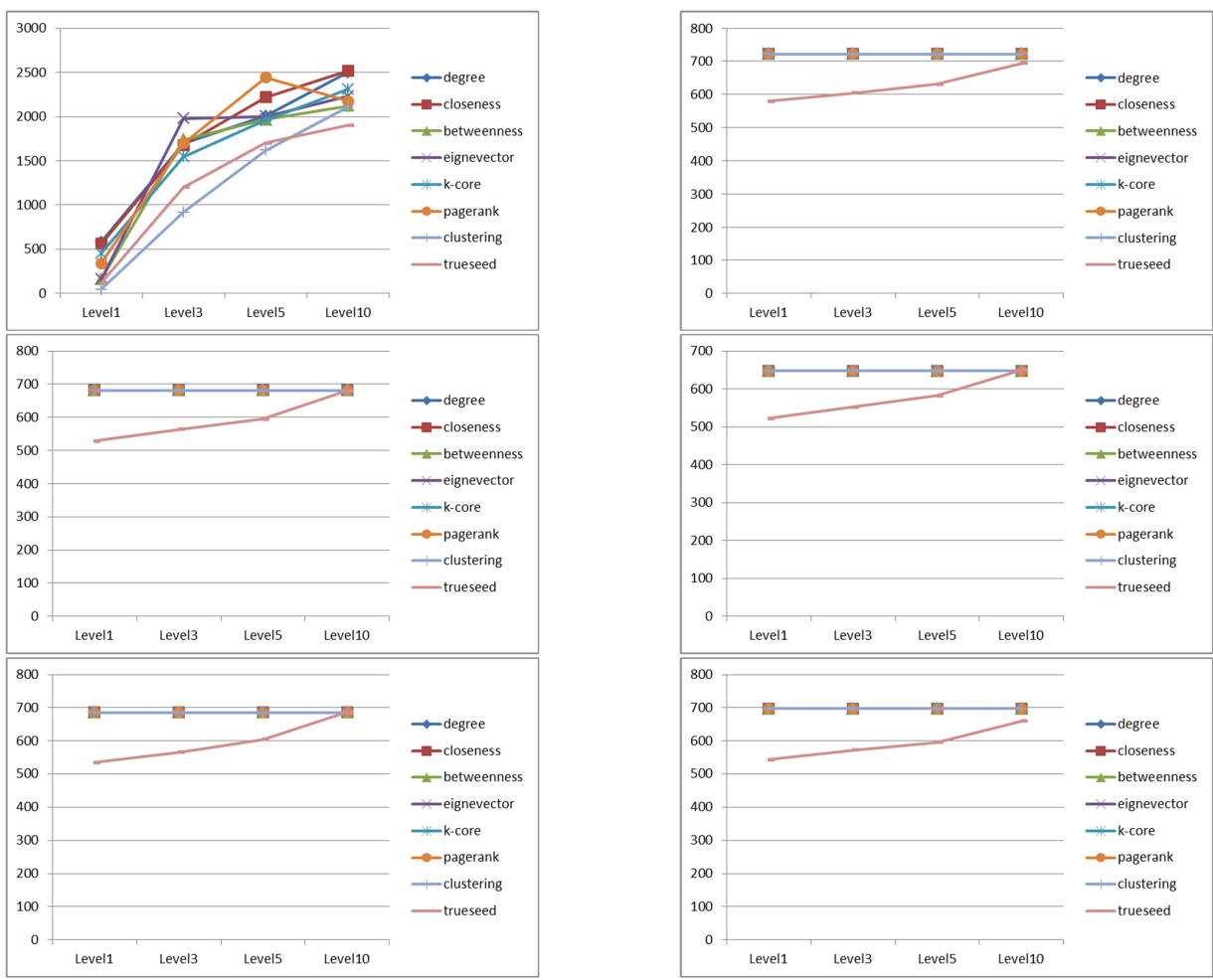

Fig. 10. Comparison of performance of centrality measures with true seeds for the SIR model with keywords \#Brexit, \#covid, \#pandemic, \#lockdown, \#quarantine, and \#mask respectively from top left to right, beginning with the first row.

From Table 8, it can be seen that although Closeness centrality and degree centrality seem to be better than other centrality measures in the initial stages of propagation, with a higher number of hops taken in consideration, the values saturate for almost all centrality measures. Overall, clustering coefficient is the weakest method for seed selection for maximization of information propagation. It can also be noted that the true seeds of the network is almost always outperformed by all centrality measures, with clustering coefficient being the only exception.

Figure 10 represents the performance of centrality measures with True Seeds as the source of propagation when propagation is simulated through SIR. It is very clearly depicted from the graphs that for each \#(hashtag), all centrality measures show better influence than true seed in terms of affected nodes.

Figure 11 represents the extent of information propagation for the top five centrality measures. It can be seen from the figure that even though initially the propagation might vary, they saturate to a similar value after five hops.

\subsection{Analysis}

For the experiment, six datasets of streamed tweets have been considered, one each for \#Brexit, \#covid, \#pandemic, \#quarantine, \#lockdown, and \#mask. From the datasets, nodes with the highest values for seven centrality measures are collected. Four information propagation models are used to simulate flow of information in the network. In Table 5, Table 6, Table 7, and Table 8, the affected nodes and levels of propagation for $\mathrm{C} 1, \mathrm{C} 2$, and $\mathrm{C} 3$ centrality values as the seed node are 

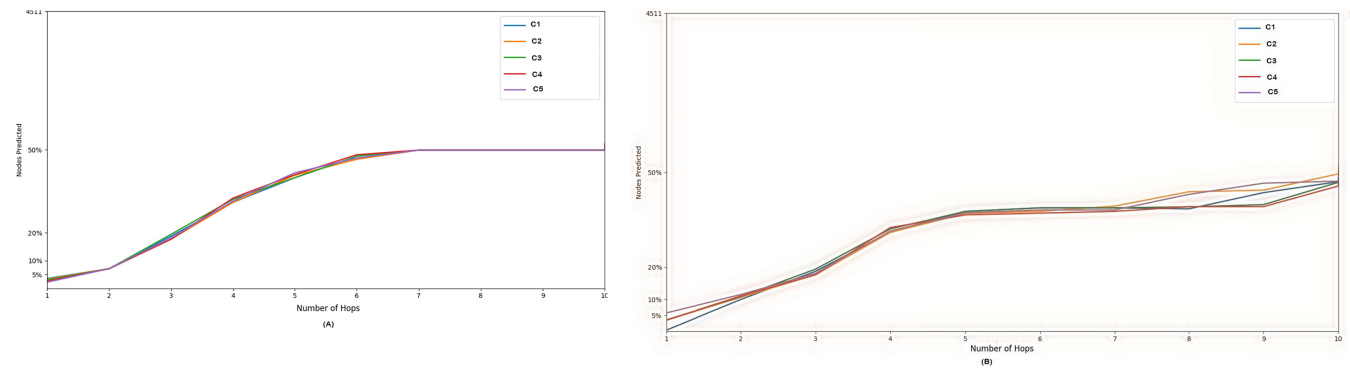

Fig. 11. Comparative graph of information propagation using the SIR model for hashtag(\#) Brexit with seeds displaying the highest Eigenvector Centrality (a) and Betweenness Centrality (b).

represented. For all five influential seeds (top five centrality measures), the propagation through graphs is shown in Figure 5, Figure 7, Figure 9, and Figure 11 for \#Brexit. A comparative study of similarity measures between centrality measures and actual source node of the information is shown in Table 4. Comparison between centrality measures along with true seed as the source of propagation are represented in Figure 4, Figure 6, Figure 8, and Figure 10.

A few interesting observations can be made from the empirical results. The graphs to compare the similarity between influential seed nodes and actual seed nodes seem to suggest the following:

- Degree centrality, Betweenness centrality, and PagrRank consistently perform well irrespective of the dataset or propagation method. However, clustering coefficient consistently performs poorly for all propagation methods and datasets except for Forest Fire. Therefore, there is an inherent handicap in clustering coefficient as influential seed selection.

- It is also evident that the choice of similarity metric used to report the results is completely unbiased. If a particular metric reports a higher value than another, it does so consistently.

- It can be seen that except for clustering coefficient, all other centrality measures perform better than true seeds detected by the two-phase algorithm in maximizing information propagation consistently irrespective of the dataset or propagation method. For forest fire, even clustering coefficient outperforms the true seeds.

The graphs to compare the extent of information propagation across different centrality nodes and different propagation algorithms seem to indicate the following:

- The differences between the extent of propagation between the $n^{\text {th }}$ selections are more starkly visible in Breadth First, Forest Fire, and SIR propagation. This seems to suggest that the Random Walk method of propagation undermines the selection of the seed node. It is thus not particularly useful. It is noticeable that Random Walk requires a lot of steps to achieve the same degree of propagation, which further tends to even out the differences that arise from seed selection. Thus, generalizations are formulated based on the other methods.

- Regardless of the method of propagation, the trend of the graph tends to remain the samethat is, all graphs uniformly have a sigmoidal shape.

- In most of the cases, the node with highest centrality enjoys a head start in terms of the extent of propagation. However, the other top $n$ selections asymptotically catch up in approximately six hops.

- In the cases corresponding to the true seeds, it can be noticed that top $n$ selections sometimes perform better than the first seed. This seems to suggest that artificial seeds selection methods using centrality measures tend to work better with the propagation techniques than natural seed selection. This indicates that the methods of seed selection through centrality measures show more potential use than actually determining the true seeds. 
From the experimental results, it can be seen that while in the initial stages of propagation, some centrality measure might outperform others, and some centrality measures might perform consistently well. However, after about five hops, the number of nodes reached by all centrality measures saturate to similar values. This can be partially attributed to the small world properties of OSNs.

From the experimental results, it can also be seen that for every keyword, when propagation begins with influential nodes selected from Node degree or PageRank as seed nodes, it spreads better to begin with. If propagation is initiated with an influential node derived from betweenness centrality as seed nodes, it begins slowly but shows a large number of affected nodes in the long run. Influential seeds measured by Closeness, Eigenvector, and K-core centrality exhibit consistent results in all cases. Seeds determined by clustering coefficient show the poorest results for all keywords and all simulated methods of propagation except Forest Fire.

\section{CONCLUSION}

A debate in information propagation theory is regarding the impact of centrality in determining seed nodes of the propagation. The main objective of this work is to thoroughly analyze the correlation between existing algorithms for seed node detection using multiple centrality measures and the flow of information in real-life social media. Finding centrality of a large network is a timeand resource-consuming problem, and although many researchers have proposed different centrality measures for scale-free networks, there is no uniform consensus about the most effective centrality measure.

The article presents comprehensive and extensive coverage on the effectiveness of different centrality measures, namely clustering coefficients, Node Degree centrality, Betweenness centrality, Closeness centrality, Eigenvector centrality, K-core centrality, and PageRank centrality for influential seed node detection. Four information propagation techniques-Breadth First Search, Random Walk, SIR, and Forest Fire-are used for simulating propagation. Seed node selection plays an important role in influence maximization, as it often offers a head start, which can determine the maximum number of affected nodes.

The proposed approach is tested on real-life Twitter data, and the algorithms are implemented on the Spark distributed computing framework. The empirical results show Betweenness centrality to be an effective seed node selecting parameter in the initial stages of propagation. In addition, degree centrality, Eigenvector, K-core, PageRank, and Closeness centrality are good choices for maximizing overall reach of information. The other centrality measure clustering coefficient has little to no effect in maximizing information propagation over the whole network in the long run.

For the propagation approaches, Random Walk suffers with the local clustering in the network. Therefore, for the approximation of the influence maximization, it is required to use modified versions of Random Walk. It can be observed that the maximum saturation level reaches approximately $20 \%$ of the total nodes, whereas for other simulations it reached almost $50 \%$ of the total population. Another important observation of the study is that in the long run, the influence of actual seeds coincides with that of centrality nodes. Therefore, centrality nodes play pivotal roles in influence maximization at the initial stages of information propagation. Whatever may be the initial seed nodes, after a certain number of hops, it reaches a threshold value and cannot spread further. In future work, an attempt to study the influential propagator to maximize information propagation in social networks will be made. An interesting observation derived from the analysis is that whatever may be the seed nodes and influence maxima, the levels are saturated within five to six hops in the network. As scale-free networks can be considered as the collection of small world networks, choosing centrality nodes as the seed node only ensures maximum propagation within the small world network. 


\section{REFERENCES}

[1] Albert-László Barabási and Réka Albert. 1999. Emergence of scaling in random networks. Science 286, 5439 (Oct. 1999), 509-512. https://doi.org/10.1126/science.286.5439.509

[2] S. Boccaletti, V. Latora, Y. Moreno, M. Chavez, and D. Hwang. 2006. Complex networks: Structure and dynamics. Physics Reports 424, 4-5 (Feb. 2006), 175-308. https://doi.org/10.1016/j.physrep.2005.10.009

[3] Ulrik Brandes. 2008. On variants of shortest-path betweenness centrality and their generic computation. Social Networks 30, 2 (May 2008), 136-145. https://doi.org/10.1016/j.socnet.2007.11.001

[4] Sung-Hyuk Cha. 2007. Comprehensive survey on distance/similarity measures between probability density functions. International fournal of Mathematical Models and Methods in Applied Sciences 1, 4 (Oct. 2007), 300-307. https://doi.org/ 10.1.1.154.8446

[5] Wei Chen and Shang-Hua Teng. 2017. Interplay between social influence and network centrality: A comparative study on Shapley centrality and single-node-influence centrality. In Proceedings of the International World Wide Web Conference (WWW'17). ACM, New York, NY, 967-976. https://doi.org/10.1145/3038912.3052608

[6] W. Chen, C. Wang, and Y. Wang. 2010. Scalable influence maximization for prevalent viral marketing in large-scale social networks. In Proceedings of the 16th ACM SIGKDD International Conference on Knowledge Discovery and Data Mining (KDD’10). ACM, New York, NY, 1029-1038. https://doi.org/10.1145/1835804.1835934

[7] Luciano da Fontoura Costa, Osvaldo N. Oliveira Jr., Gonzalo Travieso, Francisco Aparecido Rodrigues, Paulino Ribeiro Villas Boas, Lucas Antiqueira, Matheus Palhares Viana, and Luis Enrique Correa Rocha. 2011. Analyzing and modeling real-world phenomena with complex networks: A survey of applications. Advances in Physics 60, 3 (June 2011), 329412. https://doi.org/10.1080/00018732.2011.572452

[8] Paramita Dey and Sarbani Roy. 2016. Social network analysis. In Advanced Methods for Complex Network Analysis, Natarajan Meghanathan (Ed.). IGI, Hershey, PA, 237-265. https://doi.org/10.4018/978-1-4666-9964-9.ch010

[9] I. P. Erdos and A. Renyi. 1959. On random graphs. Mathematicae 6, 1 (March 1959), 290-297. http://snap.stanford.edu/ class/cs224w-readings/erdos59random.pdf.

[10] Guilherme Ferraz de Arruda, Andre Luiz Barbieri, Pablo Martin Rodriguez, Francisco A. Rodrigues, Yamir Moreno, and Luciano da Fontoura Costa. 2014. Role of centrality for the identification of influential spreaders in complex networks. Physical Review E 90, 3 (Sept. 2014), 032812. https://doi.org/10.1103/PhysRevE.90.032812

[11] Wayne Goddard and Ortrud R. Oellermann. 2010. Distance in graphs. In Structural Analysis of Complex Networks, M. Dehmer (Ed.). Springer, Boston, MA, 49-72. https://doi.org/10.1007/978-0-8176-4789-6_3

[12] Giovanna Miritello, Esteban Moro, and Rubén Lara. 2010. The dynamical strength of social ties in information spreading. Physics Review E 83, 4 (April 2010), 045102. https://doi.org/10.1103/PhysRevE.83.045102

[13] Felipe Grando and Luis C. Lamb. 2018. Computing vertex centrality measures in massive real networks with a neural learning model. In Proceedings of the International foint Conference on Neural Networks (IfCNN'18). IEEE, Los Alamitos, CA, 1-8. https://doi.org/10.1109/IJCNN.2018.8489690

[14] F. Grando, D. Noble, and L. C. Lamb. 2016. An analysis of centrality measures for complex and social networks. In Proceedings of the IEEE Global Communications Conference (GLOBECOM'16). IEEE, Los Alamitos, CA, 1-6. https://doi. org/10.1109/GLOCOM.2016.7841580

[15] R. Grassi, F. Calderoni, M. Bianchi, and A. Torriero. 2019. Betweenness to assess leaders in criminal networks: New evidence using the dual projection approach. Social Networks 56 (Jan. 2019), 23-32. https://doi.org/10.1016/j.socnet. 2018.08.001

[16] J. D. Guzman, R. F. Deckro, M. J. Robbins, J. F. Morris, and N. A. Ballester. 2014. An analytical comparison of social network measures. IEEE Transactions on Computational Social Systems 1, 1 (March 2014), 35-45. https://doi.org/10 1109/TCSS.2014.2307451

[17] J. B. Holthoefer and Y. Moreno. 2012. Absence of influential spreaders in rumor dynamics. Physical Review E 85 (Feb. 2012), 026116. https://doi.org/10.1103/PhysRevE.85.026116 PMID: 22463288.

[18] Fuad Jamour, Spiros Skiadopoulos, and Panos Kalnis. 2018. Parallel algorithm for incremental betweenness centrality on large graphs. IEEE Transactions on Parallel and Distributed Systems 29, 3 (March 2018), 659-672. https://doi.org/10 1109/TPDS.2017.2763951

[19] Saehan Jo, Jaemin Yoo, and U. Kang. 2018. Fast and scalable distributed loopy belief propagation on real-world graphs. In Proceedings of the 11th ACM International Conference on Web Search and Data Mining (WSDM'18). ACM, New York, NY, 297-305. https://doi.org/10.1145/3159652.3159722

[20] David Kempe, Jon Kleinberg, and Eva Tardos. 2003. Maximizing the spread of influence through a social network. In Proceedings of the 9th ACM SIGKDD International Conference on Knowledge Discovery and Data Mining (KDD'03). ACM, New York, NY, 137-146. https://doi.org/10.1145/956750.956769

[21] Haewoon Kwak, Changhyun Lee, Hosung Park, and Sue Moon. 2010. What is Twitter, a social network or a news media? In Proceedings of the 19th International Conference on the World Wide Web (WWW'10). ACM, New York, NY, 591-600. https://doi.org/10.1145/1772690.1772751 
[22] Lejun Zhang, Hongjie Li, Chunhai Zhao, and Xiaoying Lei. 2017. Social network information propagation model based on individual behavior. China Communications 14, 7 (Aug. 2017), 1-15. https://doi.org/10.1109/CC.2017.8010980.

[23] J. Leskovec, L. A. Adamic, and B. A. Huberman. 2007. The dynamics of viral marketing. ACM Transactions on the Web 1, 1 (May 2007), 016128. https://doi.org/10.1145/1232722.1232727

[24] Hui Li. 2018. Centrality analysis of online social network big data. In Proceedings of the IEEE 3rd International Conference on Big Data Analysis (ICBDA'18). IEEE, Los Alamitos, CA, 38-42. https://doi.org/10.1109/ICBDA.2018.8367648

[25] Matei Zaharia, Mosharaf Chowdhury, Michael J. Franklin, Scott Shenker, and Ion Stoica. 2010. Spark: Cluster computing with working sets. In Proceedings of the 2nd USENIX Conference on Hot Topics in Cloud Computing (HotCloud'10). ACM, New York, NY, 10. https://doi.org/10.5555/1863103.1863113

[26] M. Mendoza, B. Poblete, and C. Castillo. 2010. Twitter under crisis: Can we trust what we get? In Proceedings of the IEEE Global Communications Conference (SOMA'10). ACM, New York, NY, 71-79. https://doi.org/10.1145/1964858.1964869

[27] Felipe Neves, Victor Ströele, and Fernanda Campos. 2019. Information diffusion in social networks: A recommendation model in the educational context. In Proceedings of XV Brazilian Symposium on Information Systems (SBSI'19). ACM, New York, NY, 1-8. https://doi.org/10.1145/3330204.3330234

[28] M. E. Newman. 2002. Spread of epidemic disease on networks. Physical review E 66, 1 (July 2002), 016128. https: //doi.org/10.1103/PhysRevE.66.016128

[29] Lawrence Page, Sergey Brin, Rajeev Motwani, and Terry Winograd. 1999. The PageRank Citation Ranking: Bringing Order to the Web. Technical Report. Stanford InfoLab.

[30] Paramita Dey, Agneet Chaterjee, and Sarbani Roy. 2019. Influence maximization in online social network using different centrality measures as seed node of information propagation. Sadhana 44, 205 (Sept. 2019), Article 205. https://doi.org/10.1007/s12046-019-1189-7

[31] Paramita Dey, Saikat pyne, and Sarbani Roy. 2017. Information spreading in online social networks: A case study on twitter network. In Proceedings of the 18th ACM International Symposium on Mobile Ad Hoc Networking and Computing (MobiHoc'17). ACM, New York, NY, 1-2. https://doi.org/10.1145/3084041.3084071

[32] Agnieszka Rusinowska, Rudolf Berghammer, Harrie De Swart, and Michel Grabisch. 2011. Social networks: Prestige, centrality, and influence. In Proceedings of International Conference on Relational and Algebraic Methods in Computer Science (RAMICS'11). 22-39. https://doi.org/10.1007/978-3-642-21070-9_2 Invited Paper.

[33] Jimeng Sun and Jie Tang. 2011. A survey of models and algorithms for social influence analysis. In Social Network Data Analytics, C. Aggarwal (Ed.). Springer-Verlag, Boston, MA, 177-214. https://doi.org/10.1007/978-1-4419-8462-3_7

[34] R. M. Tripathy, A. Bagchi, and S. Mehta. 2010. A study of rumor control strategies on social networks. In Proceedings of the 19th ACM International Conference on Information and Knowledge Management (CIKM'10). ACM, New York, NY, 1817-1820. https://doi.org/10.1145/1871437.1871737

[35] M. P. Viana, J. L. Batista, and L. D. F. Costa. 2012. Effective number of accessed nodes in complex networks. Physical Review E 85, 036105 (Mar. 2012), 036105. https://doi.org/10.1103/PhysRevE.85.036105

[36] Michela Del Vicario, Fabiana Zollo, Guido Caldarelli, Antonio Scala, and Walter Quattrociocchi. 2017. Mapping social dynamics on Facebook: The Brexit debate. Social Networks 50, 1 (July 2017), 6-16. https://doi.org/10.1016/j.socnet.2017. 02.002

[37] Duncan J. Watts and Steven H. Strogatz. 1998. Collective dynamics of 'small-world' networks. Nature 393 (June 1998), 440-442. https://doi.org/10.1038/30918

[38] Q. Yao, R. Shi, C. Zhou, P. Wang, and L. Guo. 2015. Topic-aware social influence minimization. In Proceedings of the 24th International Conference on World Wide Web (WWW'15). ACM, New York, NY, 139-140. https://doi.org/10.1145/ 2740908.2742767

[39] Kan Chen Bak Per and Chao Tang. 1990. A forest-fire model and some thoughts on turbulence. Physics Letters A 147, 5-6 (1990), 297-300. DOI : https://doi.org/10.1016/0375-9601(90)90451-S

Received April 2020; revised August 2020; accepted May 2021 